\title{
Towards the fractional quantum Hall effect: a noncommutative geometry perspective
}

\author{
Matilde Marcolli and Varghese Mathai
}

In this paper we give a survey of some models of the integer and fractional quantum Hall effect based on noncommutative geometry. We begin by recalling some classical geometry of electrons in solids and the passage to noncommutative geometry produced by the presence of a magnetic field. We recall how one can obtain this way a single electron model of the integer quantum Hall effect. While in the case of the integer quantum Hall effect the underlying geometry is Euclidean, we then discuss a model of the fractional quantum Hall effect, which is based on hyperbolic geometry simulating the multi-electron interactions. We derive the fractional values of the Hall conductance as integer multiples of orbifold Euler characteristics. We compare the results with experimental data.

\section{Electrons in solids - Bloch theory and algebraic geometry}

We first recall some general facts about the mathematical theory of electrons in solids. In particular, after reviewing some basic facts about Bloch theory, we recall an approach pioneered by Gieseker at al. 16 [17, which uses algebraic geometry to treat the inverse problem of determining the pseudopotential from the data of the electric and optical properties of the solid.

Crystals. The Bravais lattice of a crystal is a lattice $\Gamma \subset \mathbb{R}^{d}$ (where we assume $d=2,3)$, which describes the symmetries of the crystal.

The electron-ions interaction is described by a periodic potential

$$
U(x)=\sum_{\gamma \in \Gamma} u(x-\gamma),
$$

namely, $U$ is invariant under the translations in $\Gamma$,

$$
T_{\gamma} U=U, \quad \forall \gamma \in \Gamma .
$$

When one takes into account the mutual interaction of electrons, one obtains an $N$-particles Hamiltonian of the form

$$
\sum_{i=1}^{N}\left(-\Delta_{x_{i}}+U\left(x_{i}\right)\right)+\frac{1}{2} \sum_{i \neq j} W\left(x_{i}-x_{j}\right) .
$$


This can be treated in the independent electron approximation, namely by introducing a modification $V$ of the single electron potential

$$
\sum_{i=1}^{N}\left(-\Delta_{x_{i}}+V\left(x_{i}\right)\right)
$$

It is remarkable that, even though the original potential $U$ is unbounded, a reasonable independent electron approximation can be obtained with $V$ a bounded function.

The wave function for the $N$-particle problem (1.4) is then of the form

$$
\psi\left(x_{1}, \ldots, x_{N}\right)=\operatorname{det}\left(\psi_{i}\left(x_{j}\right)\right),
$$

for $(-\Delta+V) \psi_{i}=E_{i} \psi_{i}$ so that $\sum\left(-\Delta_{x_{i}}+V\left(x_{i}\right)\right) \psi=E \psi$ with $E=\sum E_{i}$. This reduces a multi-electron problem to the single particle case.

However, in this approximation, usually the single electron potential $V$ is not known explicitly, hence the focus shifts on the inverse problem of determining $V$.

Bloch electrons. Let $T_{\gamma}$ denote the unitary operator on $\mathcal{H}=L^{2}\left(\mathbb{R}^{d}\right)$ implementing the translation by $\gamma \in \Gamma$, as in (1.2). We have, for $H=-\Delta+V$,

$$
T_{\gamma} H T_{\gamma^{-1}}=H, \quad \forall \gamma \in \Gamma .
$$

Thus, we can simultaneously diagonalize these operators. This can be done via characters of $\Gamma$, or equivalently, via its Pontrjagin dual $\hat{\Gamma}$. In fact, the eigenvalue equation is of the form $T_{\gamma} \psi=c(\gamma) \psi$. Since $T_{\gamma_{1} \gamma_{2}}=T_{\gamma_{1}} T_{\gamma_{2}}$, and the operators are unitaries, we have $c: \Gamma \rightarrow U(1)$, of the form

$$
c(\gamma)=e^{i\langle k, \gamma\rangle}, \quad k \in \hat{\Gamma} .
$$

The Pontrjagin dual $\hat{\Gamma}$ of the abelian group $\Gamma \cong \mathbb{Z}^{d}$ is a compact group isomorphic to $T^{d}$, obtained by taking the dual of $\mathbb{R}^{d}$ modulo the reciprocal lattice

$$
\Gamma^{\sharp}=\left\{k \in \mathbb{R}^{d}:\langle k, \gamma\rangle \in 2 \pi \mathbb{Z}, \forall \gamma \in \Gamma\right\} .
$$

Brillouin zones. By definition, the Brillouin zones of a crystals are fundamental domains for the reciprocal lattice $\Gamma^{\sharp}$ obtained via the following inductive procedure. The Bragg hyperplanes of a crystal are the hyperplanes along which a pattern of diffraction of maximal intensity is observed when a beam of radiation (X-rays for instance) is shone at the crystal. The $N$-th Brillouin zone consists of all the points in (the dual) $\mathbb{R}^{d}$ such that the line from that point to the origin crosses exactly $(n-1)$ Bragg hyperplanes of the crystal. 


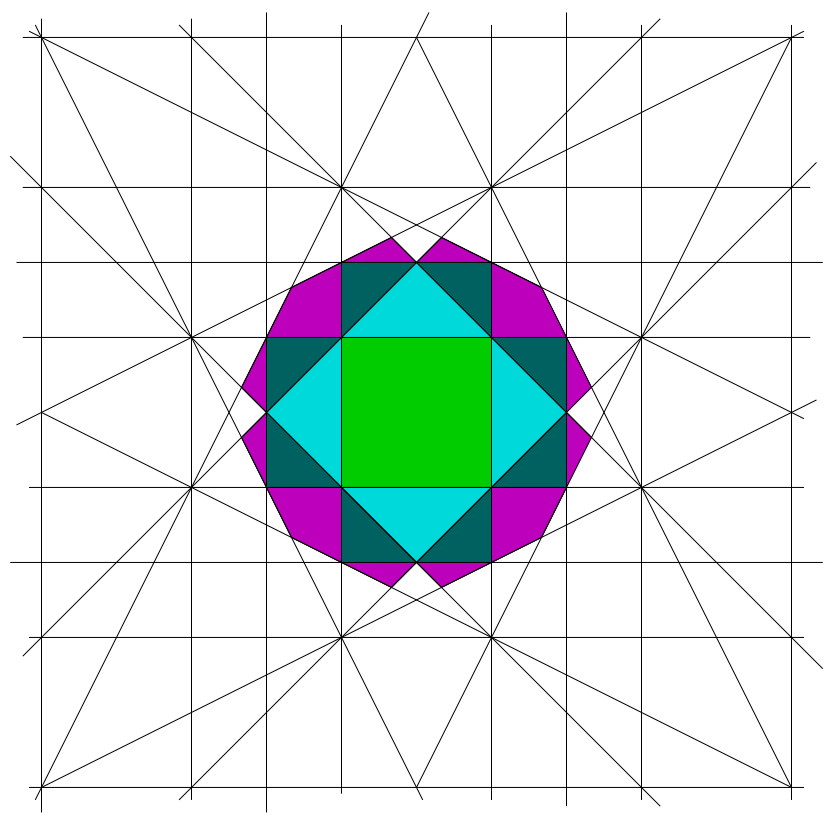

Figure 1. Brillouin zones in a 2D crystal

Band structure. One obtains this way ( $c f$. [16 ) a family self-adjoint elliptic boundary value problems, parameterized by the lattice momenta $k \in \mathbb{R}^{d}$,

$$
D_{k}=\left\{\begin{array}{c}
(-\Delta+V) \psi=E \psi \\
\psi(x+\gamma)=e^{i\langle k, \gamma\rangle} \psi(x)
\end{array}\right.
$$

For each value of the momentum $k$, one has eigenvalues $\left\{E_{1}(k), E_{2}(k), E_{3}(k), \ldots\right\}$. As functions of $k$, these satisfy the periodicity

$$
E(k)=E(k+u) \quad \forall u \in \Gamma^{\sharp} .
$$

It is customary therefore to plot the eigenvalue $E_{n}(k)$ over the n-the Brillouin zone and obtain this way a map

$$
k \mapsto E(k) \quad k \in \mathbb{R}^{d}
$$

called the energy-crystal momentum dispersion relation.

Fermi surfaces and complex geometry. Many electric and optical properties of the solid can be read off the geometry of the Fermi surface. This is a hypersurface $F$ in the space of crystal momenta $k$,

$$
F_{\lambda}(\mathbb{R})=\left\{k \in \mathbb{R}^{d}: E(k)=\lambda\right\} .
$$

A comprehensive archive of Fermi surfaces for various chemical elements can be found in [9], or online at http://www.phys.ufl.edu/fermisurface We reproduce in Figure 2 an example of the complicated geometry of Fermi surfaces.

The approach to the theory of electrons in solids proposed by [16 [17 is based on the idea that the geometry of the Fermi surfaces can be better understood by passing 


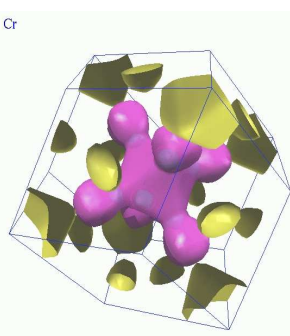

Chromium

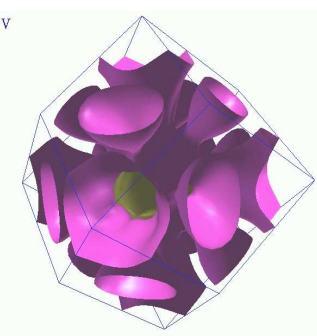

Vanadium

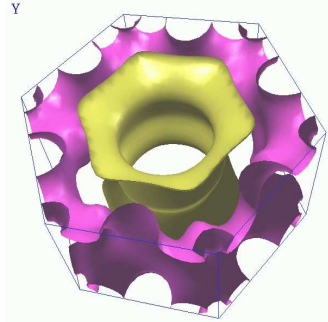

Yttrium

Figure 2. Examples of Fermi surfaces

to complex geometry and realizing (1.8) as a cycle on a complex hypersurface. One considers first the complex Bloch variety defined by the condition

$$
B(V)=\left\{(k, \lambda) \in \mathbb{C}^{d+1}: \begin{array}{cc}
\exists \psi & \text { nontrivial solution of } \\
& (-\Delta+V) \psi=\lambda \psi \\
& \psi(x+\gamma)=e^{i\langle k, \gamma\rangle} \psi(x)
\end{array}\right\}
$$

Then the complex Fermi surfaces are given by the fibers of the projection to $\lambda \in \mathbb{C}$,

$$
F_{\lambda}(\mathbb{C})=\pi^{-1}(\lambda) \subset B(V) .
$$

This is a complex hypersurface in $\mathbb{C}^{d}$. To apply the tools of projective algebraic geometry, one works in fact with a singular projective hypersurface (a compactification of $B(V)$, cf. [16]).

One can then realize the original Fermi surface (1.8) as a cycle $F_{\lambda} \cap \mathbb{R}^{d}=F_{\lambda}(\mathbb{R})$ representing a class in the homology $H_{d-1}\left(F_{\lambda}(\mathbb{C}), \mathbb{Z}\right)$. A result of $[\mathbf{1 6}$ is that the integrated density of states

$$
\rho(\lambda)=\lim _{\ell \rightarrow \infty} \frac{1}{\ell} \#\{\text { eigenv of } H \leq \lambda\},
$$

for $H=-\Delta+V$ on $L^{2}\left(\mathbb{R}^{d} / \ell \Gamma\right)$, is obtained from a period

$$
\frac{d \rho}{d \lambda}=\int_{F_{\lambda}(\mathbb{R})} \omega_{\lambda}
$$

where $\omega_{\lambda}$ is a holomorphic differential on $F_{\lambda}(\mathbb{C})$.

Discretization. It is often convenient to treat problems like (1.7) by passing to a discretized model. On $\ell^{2}\left(\mathbb{Z}^{d}\right)$, one considers the random walk operator

$$
\begin{aligned}
\mathcal{R} \psi\left(n_{1}, \ldots, n_{d}\right)= & +\sum_{i=1}^{d} \psi\left(n_{1}, \ldots, n_{i}+1, \ldots, n_{d}\right) \\
& +\sum_{i=1}^{d} \psi\left(n_{1}, \ldots, n_{i}-1, \ldots, n_{d}\right) .
\end{aligned}
$$

This is related to the discretized Laplacian by

$$
\Delta \psi\left(n_{1}, \ldots, n_{d}\right)=(2 d-\mathcal{R}) \psi\left(n_{1}, \ldots, n_{d}\right)
$$


In this discretization, the complex Bloch variety is described by a polynomial equation in $z_{i}, z_{i}^{-1}$ (cf. [16])

$$
B(V)=\left\{\left(z_{1} \ldots, z_{d}, \lambda\right): \begin{array}{l}
\exists \psi \in \ell^{2}(\Gamma) \text { nontriv sol of } \\
R_{\gamma_{i}} \psi=z_{i} \psi
\end{array}\right\},
$$

where $R_{\gamma_{i}} \psi\left(n_{1}, \ldots, n_{d}\right)=\psi\left(n_{1}, \ldots, n_{i}+a_{i}, \ldots, n_{d}\right)$.

It will be very useful for us in the following to also consider a more general random walk for a discrete group $\Gamma$, as an operator on $\mathcal{H}=\ell^{2}(\Gamma)$. Let $\gamma_{i}$ be a symmetric set of generators of $\Gamma$, i.e. a set of generators and their inverses. The random walk operator is defined as

$$
\mathcal{R} \psi(\gamma)=\sum_{i=1}^{r} R_{\gamma_{i}} \psi(\gamma)=\sum_{i=1}^{r} \psi\left(\gamma \gamma_{i}\right)
$$

and the corresponding discretized Laplacian is $\Delta=r-\mathcal{R}$.

The breakdown of classical Bloch theory. The approach to the study of electrons in solids via Bloch theory breaks down when either a magnetic field is present, or when the periodicity of the lattice is replaced by an aperiodic configuration, such as those arising in quasi-crystals. What is common to both cases is that the commutation relation $T_{\gamma} H=H T_{\gamma}$ fails.

Both cases can be studied by replacing ordinary geometry by noncommutative geometry (cf. 12 ). Ordinary Bloch theory is replaced by noncommutative Bloch theory [18. A good introduction to the treatment via noncommutative geometry of the case of aperiodic solids can be found in $[$.

For our purposes, we are mostly interested in the other case, namely the presence of magnetic field, as that is the source of the Hall effects. Bellissard pioneered an approach to the quantum Hall effect via noncommutative geometry and derived a complete and detailed mathematical model for the Integer Quantum Hall Effect within this framework, $\mathbf{3}$.

\section{Quantum Hall Effect}

We describe the main aspects of the classical and quantum (integer and fractional) Hall effects, and some of the current approaches used to produce a mathematical model. Our introduction will not be exhaustive. In fact, for reasons of space, we will not discuss many interesting mathematical results on the quantum Hall effect and will concentrate mostly on the direction leading to the use of noncommutative geometry.

Classical Hall effect. The classical Hall effect was first observed in the XIX century [19. A thin metal sample is immersed in a constant uniform strong orthogonal magnetic field, and a constant current $\mathbf{j}$ flows through the sample, say, in the $x$-direction. By Flemming's rule, an electric field is created in the $y$-direction, as the flow of charge carriers in the metal is subject to a Lorentz force perpendicular to the current and the magnetic field. This is called the Hall current.

The equation for the equilibrium of forces in the sample

$$
N e \mathbf{E}+\mathbf{j} \wedge \mathbf{B}=0,
$$




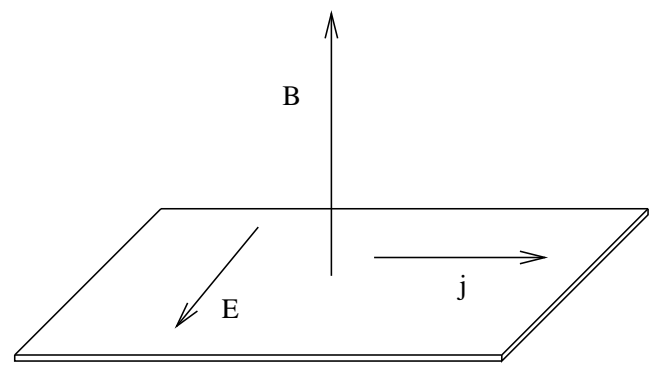

Figure 3. Hall effect

defines a linear relation. The ratio of the intensity of the Hall current to the intensity of the electric field is the Hall conductance,

$$
\sigma_{H}=\frac{N e \delta}{B} .
$$

In the stationary state, $\sigma_{H}$ is proportional to the dimensionless filling factor $\nu=\frac{\rho h}{e B}$, where $\rho$ is the 2-dimensional density of charge carriers, $h$ is the Planck constant, and $e$ is the electron charge. More precisely, we have

$$
\sigma_{H}=\frac{\nu}{R_{H}}
$$

where $R_{H}=h / e^{2}$ denotes the Hall resistance, which is a universal constant. This measures the fraction of Landau level filled by conducting electrons in the sample.

Integer quantum Hall effect. In 1980, von Klitzing's experiment showed that, lowering the temperature below $1 \mathrm{~K}$, quantum effects dominate, and the relation of Hall conductance to filling factor shows plateaux at integer values, 21]. The effect is measured with very high precision (of the order of $10^{-8}$ ) and allows for a very accurate measurement of the fine structure constant $e^{2} / \hbar c$.

Under the above conditions, one can effectively use the independent elector approximation discussed in the previous section and reduce the problem to a single particle case.

The main physical properties of the integer quantum Hall effect are the following:

- $\sigma_{H}$, as a function of $\nu$, has plateaux at integer multiples of $e^{2} / h$.

- At values of $\nu$ corresponding to the plateaux, the conductivity along the current density axis (direct conductivity) vanishes.

Laughlin first suggested that IQHE should have a geometric explanation 23. More precisely, the fact that the quantization of the Hall conductance appears as a very robust phenomenon, insensitive to changes in the sample and its geometry, or to the presence of impurities, suggests the fact that the effect should have the same qualities of the index theorem, which assigns an integer invariant to an elliptic differential operator, in a way that is topological and independent of perturbations. The prototype of such index theorems is the Gauss-Bonnet theorem, which extracts from an infinitesimally variable quantity, the curvature of a closed surface, a robust topological invariant, its Euler characteristic. The idea of modelling the integer quantum Hall effect on an index theorem started fairly early after the discovery 


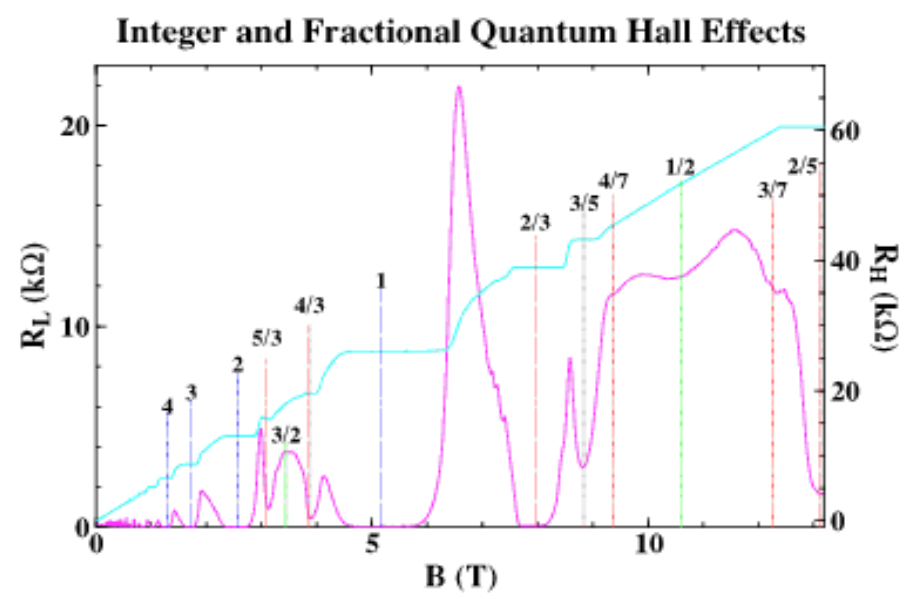

Figure 4. Fractional quantum Hall effect

of the effect. Laughlin's formulation can already be seen as a form of GaussBonnet, while this was formalized more precisely in such terms shortly afterwards by Thouless et al. (1982) and by Avron, Seiler, and Simon (1983) (cf. [31 1]).

One of the early successes of Connes' noncommutative geometry [12] was a rigorous mathematical model of the integer quantum Hall effect, developed by Bellissard, van Elst, and Schulz-Baldes, 3. Unlike the previous models, this accounts for all the aspects of the phenomenon: integer quantization, localization, insensitivity to the presence of disorder, and vanishing of direct conductivity at plateaux levels. Again the integer quantization is reduced to an index theorem, albeit of a more sophisticated nature, involving the Connes-Chern character, the $K$-theory of $C^{*}$ algebras and cyclic cohomology ( $c f$. 11]).

Fractional quantum Hall effect. The fractional QHE was discovered by Stormer and Tsui in 1982. The setup is as in the quantum Hall effect: in a high quality semi-conductor interface, which will be modelled by an infinite 2-dimensional surface, with low carrier concentration and extremely low temperatures $\sim 10 \mathrm{mK}$, in the presence of a very strong magnetic field, the experiment shows that the same graph of $\frac{h}{e^{2}} \sigma_{H}$ against the filling factor $\nu$ exhibits plateaux at certain fractional values (Figure 4).

Under the conditions of the experiments, the independent electron approximation that reduces the problem to a single electron is no longer viable and one has to incorporate the Coulomb interaction between the electrons in a many-electron theory. For this reason, many of the proposed mathematical models of the fractional quantum Hall effect resort to quantum field theory and, in particular, Chern-Simons theory (cf. e.g. [28]).

In this survey we will only discuss a proposed model 24] 25, which is based on extending the validity of the Bellissard approach to the setting of hyperbolic geometry as in [5], where passing to a negatively curved geometry is used as a 
device to simulate the many-electrons Coulomb interaction while remaining within a single electron model.

What is expected of any proposed mathematical model? Primarily three things: to account for the strong electron interactions, to exhibit the observed fractions and predict new fractions, and to account for the varying width of the observed plateaux. We will discuss these various aspects in the rest of the paper.

\section{Noncommutative geometry models}

In the theory of the quantum Hall effect noncommutativity arises from the presence of the magnetic field, which has the effect of turning the Brillouin zone into a noncommutative space. In Bellissard's model of the integer quantum Hall effect 3 the noncommutative space obtained this way is the noncommutative torus and the integer values of the Hall conductance are obtained from the corresponding Connes-Chern character. We will consider a larger class of noncommutative spaces, associated to the action of a Fuchsian group of the first kind without parabolic elements on the hyperbolic plane. The idea is that, by effect of the strong interaction with the other electrons, a single electron "sees" the surrounding geometry as curved, while the lattice sites appear to the moving electron, in a sort of multiple image effect, as sites in a lattice in the hyperbolic plane. This model will recover the integer values but will also produce fractional values of the Hall conductance.

3.1. Hyperbolic geometry. Let $\mathbb{H}$ denote the hyperbolic plane. Its geometry is described as follows. Consider the pseudosphere $\left\{x^{2}+y^{2}+z^{2}-t^{2}=1\right\}$ in 4-dimensional Minkowski space-time $M$. The $z=0$ slice of the pseudosphere realizes an isometric embedding of the hyperbolic plane $\mathbb{H}$ in $M$. In this geometry, a periodic lattice on the resulting surface is determined by a Fuchsian group $\Gamma$ of isometries of $\mathbb{H}$ of signature $\left(g ; \nu_{1}, \ldots, \nu_{n}\right)$,

$$
\Gamma=\Gamma\left(g ; \nu_{1}, \ldots, \nu_{n}\right) .
$$

This is a discrete cocompact subgroup $\Gamma \subset \operatorname{PSL}(2, \mathbb{R})$ with generators $a_{i}, b_{i}, c_{j}$, with $i=1, \ldots, g$ and $j=1, \ldots, n$ and a presentation of the form

$$
\Gamma\left(g ; \nu_{1}, \ldots, \nu_{n}\right)=\left\langle a_{i}, b_{i}, c_{j} \mid \prod_{i=1}^{g}\left[a_{i}, b_{i}\right] c_{1} \cdots c_{n}=1, \quad c_{j}^{\nu_{j}}=1\right\rangle .
$$

The quotient of the action of $\Gamma$ by isometrieson $\mathbb{H}$,

$$
\Sigma\left(g ; \nu_{1}, \ldots, \nu_{n}\right):=\Gamma \backslash \mathbb{H},
$$

is a hyperbolic orbifold, namely a compact Riemann surface of genus $g$ with $n$ cone points $\left\{x_{1}, \ldots, x_{n}\right\}$, which are the image of points in $\mathbb{H}$ with non-trivial stabilizer of the action of $\Gamma$. In the torsion free case, where we only have generators $a_{i}$ and $b_{i}$, we obtain smooth compact Riemann surfaces of genus $g$. 


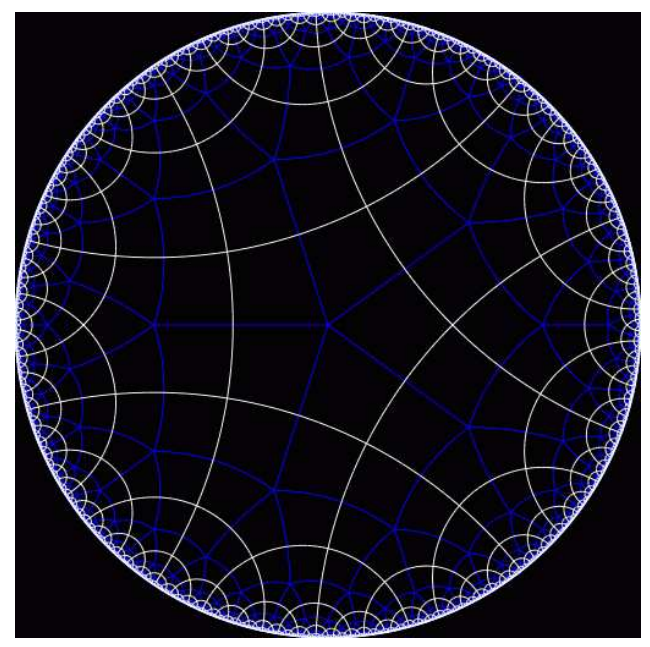

FIgURE 5. Tiling of the hyperbolic plane

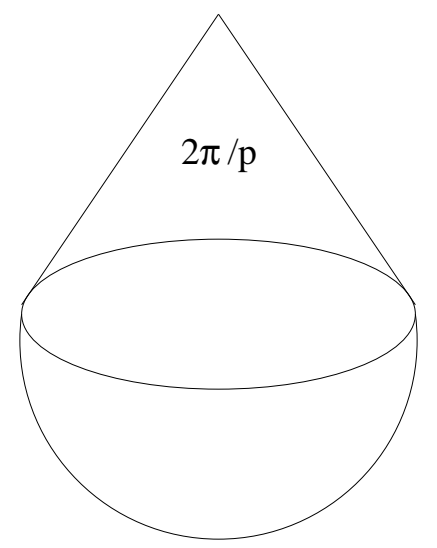

FiguRE 6. Thurston's teardrop orbifold

Orbifolds. The space $\Sigma\left(g ; \nu_{1}, \ldots, \nu_{n}\right)$ of (3.3) is a special case of good orbifolds. These are orbifolds that are orbifold-covered by a smooth manifold. In dimension two, in the oriented compact case, the only exceptions (bad orbifolds) are the Thurston teardrop (Figure 6 ) with a single cone point of angle $2 \pi / p$, and the double teardrop.

In particular, all the hyperbolic orbifolds $\Sigma\left(g ; \nu_{1}, \ldots, \nu_{n}\right)$ are good orbifolds and they are orbifold-covered by a smooth compact Riemann surface,

$$
\Sigma_{g^{\prime}} \stackrel{G}{\longrightarrow} \Sigma\left(g ; \nu_{1}, \ldots, \nu_{n}\right)=\Gamma \backslash \mathbb{H},
$$

where the genus $g^{\prime}$ satisfies the Riemann-Hurwitz formula for branched covers

$$
g^{\prime}=1+\frac{\# G}{2}(2(g-1)+(n-\nu))
$$


for $\nu=\sum_{j=1}^{n} \nu_{j}^{-1}$.

Notice moreover that the orbifolds $\Sigma=\Sigma\left(g ; \nu_{1}, \ldots, \nu_{n}\right)$ are an example of classifying space for proper actions in the sense of Baum-Connes, namely they are of the form

$$
\Sigma=\underline{B} \Gamma=\Gamma \backslash \underline{E} \Gamma .
$$

An important invariant of orbifold geometry, which will play a crucial role in our model of the fractional quantum Hall effect, is the orbifold Euler characteristic. This is an analog of the usual topological Euler characteristic, but it takes values in rational numbers, $\chi_{o r b}(\Sigma) \in \mathbb{Q}$. It is multiplicative over orbifold covers, it agrees with the usual topological Euler characteristic $\chi$ for smooth manifolds, and it satisfies the inclusion-exclusion principle

$$
\begin{aligned}
\chi_{o r b}\left(\Sigma_{1} \cup \cdots \cup \Sigma_{r}\right)= & \sum_{i} \chi_{o r b}\left(\Sigma_{i}\right)-\sum_{i, j} \chi_{o r b}\left(\Sigma_{i} \cap \Sigma_{j}\right) \\
\cdots & +(-1)^{r+1} \chi_{o r b}\left(\Sigma_{1} \cap \cdots \cap \Sigma_{r}\right) .
\end{aligned}
$$

In the case of the hyperbolic orbifolds $\Sigma\left(g ; \nu_{1}, \ldots, \nu_{n}\right)$, the orbifold Euler characteristic is given by the formula

$$
\chi_{o r b}\left(\Sigma\left(g ; \nu_{1}, \ldots, \nu_{n}\right)\right)=2-2 g+\nu-n .
$$

Magnetic field and symmetries. The magnetic field can be described by a 2 -form $\omega=d \eta$, where $\omega$ and $\eta$ are the field and potential, respectively, subject to the customary relation $\mathbf{B}=\operatorname{curl} \mathbf{A}$.

One then considers the magnetic Schrödinger operator

$$
\Delta^{\eta}+V \text {, }
$$

where the magnetic Laplacian is given by $\Delta^{\eta}:=(d-i \eta)^{*}(d-i \eta)$ and $V$ is the electric potential of the independent electron approximation.

The 2-form $\omega$ satisfies the periodicity condition $\gamma^{*} \omega=\omega$, for all $\gamma \in \Gamma=\mathbb{Z}^{d}$ (e.g. one might assume that the magnetic field is a constant field $B$ perpendicular to the sample). Thus, we have the relation $0=\omega-\gamma^{*} \omega=d\left(\eta-\gamma^{*} \eta\right)$, which implies

$$
\eta-\gamma^{*} \eta=d \phi_{\gamma} .
$$

Due to the fact that $\eta$ itself need not be periodic, but only subject to condition (3.10), the magnetic Laplacian no longer commutes with the $\Gamma$ action, unlike the ordinary Laplacian. This is, in a nutshell, how turning on a magnetic field brings about noncommutativity.

What are then the symmetries of the magnetic Laplacian? These are given by the magnetic translations. Namely, after writing (3.10) in the form $\phi_{\gamma}(x)=\int_{x_{0}}^{x}(\eta-$ $\gamma^{*} \eta$ ), we consider the unitary operators

$$
T_{\gamma}^{\phi} \psi:=\exp \left(i \phi_{\gamma}\right) T_{\gamma} \psi
$$

It is easy to check that these satisfy the desired commutativity $(d-i \eta) T_{\gamma}^{\phi}=$ $T_{\gamma}^{\phi}(d-i \eta)$. However, commutativity is still lost in another way, namely, magnetic translations, unlike the ordinary translations by elements $\gamma \in \Gamma=\mathbb{Z}^{d}$, no longer commute among themselves (except in the case of integer flux). We have instead

$$
T_{\gamma}^{\phi} T_{\gamma^{\prime}}^{\phi}=\sigma\left(\gamma, \gamma^{\prime}\right) T_{\gamma \gamma^{\prime}}^{\phi}
$$


Instead of obtaining a representation of $\Gamma$, the magnetic translations give rise to a projective representation, with the cocycle

$$
\sigma\left(\gamma, \gamma^{\prime}\right)=\exp \left(-i \phi_{\gamma}\left(\gamma^{\prime} x_{0}\right)\right)
$$

where $\phi_{\gamma}(x)+\phi_{\gamma^{\prime}}(\gamma x)-\phi_{\gamma^{\prime} \gamma}(x)$ is independent of $x$.

Algebra of observables. The $C^{*}$-algebra of observables should be minimal, yet large enough to contain all of the spectral projections onto gaps in the spectrum of the magnetic Schrödinger operators $\Delta^{\eta}+V$ for any periodic potential $V$. Now let $\mathcal{U}$ denote the set of all bounded operators on $L^{2}(\mathbb{H})$ that commute with the magnetic translations. By a theorem of von Neumann, $\mathcal{U}$ is a von Neumann algebra. By Lemma 1.1, 22. any element $Q \in \mathcal{U}$ can be represented uniquely as

$$
Q=\sum_{\gamma \in \Gamma} T_{\gamma}^{-\phi} \otimes Q(\gamma)
$$

where $Q(\gamma)$ is a bounded operator on the Hilbert space $L^{2}(\mathbb{H} / \Gamma)$. Let $\mathcal{L}^{1}$ denote the subset of $\mathcal{U}$ consisting of all bounded operators on $Q$ on $L^{2}(\mathbb{H})$ that commute with the magnetic translations and such that $\sum_{\gamma \in \Gamma}\|Q(\gamma)\|<\infty$. The norm closure of $\mathcal{L}^{1}$ is a $C^{*}$-algebra denoted by $\mathcal{C}^{*}$, that is taken to be the algebra of observables. Using the Riesz representation for projections onto spectral gaps cf.(3.26), one can show as in 22 that $C^{*}$ contains all of the projections onto the spectral gaps of the magnetic Schrödinger operators. In fact, it can be shown that $C^{*}$ is Morita equivalent to the reduced twisted group $C^{*}$ algebra $C_{r}^{*}(\Gamma, \bar{\sigma})$, explained later in the text, showing that in both the continuous and the discrete models for the quantum Hall effect, the algebra of observables are Morita equivalent, so they describe the same physics. Hence we will mainly discuss the discrete model in this paper.

Semiclassical properties, as the electro-magnetic coupling constant goes to zero. Recall the magnetic Schrödinger operator

$$
\Delta^{\eta}+\mu^{-2} V
$$

where $\Delta^{\eta}$ is the magnetic Laplacian, $V$ is the electric potential and $\mu$ is the electromagnetic coupling constant. When $V$ is a Morse type potential, i.e. for all $x \in \mathbb{H}$, $V(x) \geq 0$. Moreover, if $V\left(x_{0}\right)=0$ for some $x_{0}$ in $\mathcal{M}$, then there is a positive constant $c$ such that $V(x) \geq c\left|x-x_{0}\right|^{2} I$ for all $x$ in a neighborhood of $x_{0}$. Also assume that $V$ has at least one zero point. Observe that all functions $V=|d f|^{2}$, where $|d f|$ denotes the pointwise norm of the differential of a $\Gamma$-invariant Morse function $f$ on $\mathbb{H}$, are examples of Morse type potentials.

Under these assumptions, the semiclassical properties of the spectrum of the magnetic Schrödinger operator, and the Hall conductance were studied by Kordyukov, Mathai and Shubin in 22], as the electro-magnetic coupling constant $\mu$ goes to zero. One result obtained is that there exists an arbitrarily large number of gaps in the spectrum of the magnetic Schrödinger operator for all $\mu$ sufficiently small. Another result obtained in [22] is that the low energy bands do not contribute to the Hall conductance, again for all $\mu$ sufficiently small. 
Extending Pontrjagin duality. An advantage of noncommutative geometry is that it provides a natural generalization of Pontrjagin duality. Namely, the duals of discrete groups are noncommutative spaces.

In fact, first recall that, if $\Gamma$ is a discrete abelian group, then its Pontrjagin dual $\hat{\Gamma}$, which is the group of characters of $\Gamma$ is a compact abelian group. The duality is given by Fourier transform $e^{i\langle k, \gamma\rangle}$, for $\gamma \in \Gamma$ and $k \in \hat{\Gamma}$.

In particular, this shows that the algebra of functions on $\hat{\Gamma}$ can be identified with the (reduced) $C^{*}$-algebra of the group $\Gamma$,

$$
C(\hat{\Gamma}) \cong C_{r}^{*}(\Gamma),
$$

where the reduced $C^{*}$-algebra $C_{r}^{*}(\Gamma)$ is the $C^{*}$-algebra generated by $\Gamma$ in the regular representation on $\ell^{2}(\Gamma)$.

When $\Gamma$ is non-abelian, although Pontrjagin duality no longer works in the classical sense, the left hand side of (3.15) still makes perfect sense and behaves "like" the algebra of functions on the dual group. In other words, we can say that, for a non-abelian group, the Pontrjagin dual $\hat{\Gamma}$ still exists as a noncommutative space whose algebra of functions is $C_{r}^{*}(\Gamma)$.

This point of view can be adopted to work with the theory of electrons in solids whenever classical Bloch theory breaks down. In the case of aperiodicity, the dual $\hat{\Gamma}$ (which is identified with the Brillouin zone) is replaced by a noncommutative $C^{*}$ algebra. This is the case, similarly, for the presence of magnetic field in the quantum Hall effect. The magnetic field deforms the Brillouin zone to a noncommutative space, given by the (noncommutative) algebra of the magnetic translation.

Harper operators. It is again convenient to discretize the problem. The discretized magnetic Laplacian is given in terms of the Harper operator, which is an analog of the random walk operator seen in the previous section, but defined using the magnetic translations. For $\Gamma=\mathbb{Z}^{2}$, the Harper operator is of the form

$$
\begin{array}{rlr}
H_{\alpha_{1}, \alpha_{2}} \psi(m, n) & =e^{-i \alpha_{1} n} & \psi(m+1, n) \\
& +e^{i \alpha_{1} n} & \psi(m-1, n) \\
& +e^{-i \alpha_{2} m} & \psi(m, n+1) \\
& +e^{i \alpha_{2} m} & \psi(m, n-1) .
\end{array}
$$

Here the 2-cocycle $\sigma: \Gamma \times \Gamma \rightarrow U(1)$ is given by

$$
\sigma\left(\left(m^{\prime}, n^{\prime}\right),(m, n)\right)=\exp \left(-i\left(\alpha_{1} m^{\prime} n+\alpha_{2} m n^{\prime}\right)\right) .
$$

The magnetic translations are generated by $U=R_{(0,1)}^{\sigma}$ and $V=R_{(1,0)}^{\sigma}$ of the form

$$
U \psi(m, n)=\psi(m, n+1) e^{-i \alpha_{2} m} \quad V \psi(m, n)=\psi(m+1, n) e^{-i \alpha_{1} n} .
$$

These satisfy the commutation relations of the noncommutative torus $\mathcal{A}_{\theta}$, with $\theta=\alpha_{2}-\alpha_{1}$, namely

$$
U V=e^{i \theta} V U .
$$

The Harper operator (3.16) is in fact more simply written as $H_{\sigma}=U+U^{*}+V+V^{*}$. 


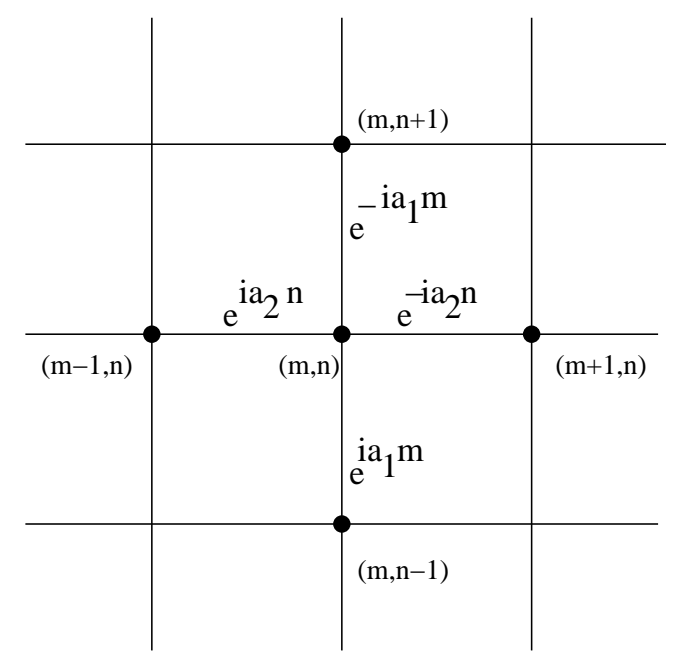

FiguRE 7. The Harper operator on the square lattice

This shows that, on a 2-dimensional lattice, the effect of the magnetic field is to deform the usual Brillouin zone (which is an ordinary torus $T^{2}$ ) to a noncommutative torus, where the parameter $\theta$ depends on the magnetic flux through a cell of the lattice.

As in the case of the discretization of the ordinary Laplacian, for the magnetic Laplacian we can also consider the corresponding Harper operator on a more general (possibly non-abelian) discrete group $\Gamma$. This will be useful later, in our model of the fractional quantum Hall effect, but we introduce it here for convenience. For the general setup for finitely generated discrete groups recalled here below, we follow [5].

Suppose given a finitely generated discrete group $\Gamma$ and a multiplier $\sigma: \Gamma \times \Gamma \rightarrow U(1)$ (a 2-cocycle)

$$
\sigma\left(\gamma_{1}, \gamma_{2}\right) \sigma\left(\gamma_{1} \gamma_{2}, \gamma_{3}\right)=\sigma\left(\gamma_{1}, \gamma_{2} \gamma_{3}\right) \sigma\left(\gamma_{2}, \gamma_{3}\right)
$$

$\sigma(\gamma, 1)=\sigma(1, \gamma)=1$

On the Hilbert space $\ell^{2}(\Gamma)$, consider the left/right $\sigma$-regular representations

$$
L_{\gamma}^{\sigma} \psi\left(\gamma^{\prime}\right)=\psi\left(\gamma^{-1} \gamma^{\prime}\right) \sigma\left(\gamma, \gamma^{-1} \gamma^{\prime}\right) \quad R_{\gamma}^{\sigma} \psi\left(\gamma^{\prime}\right)=\psi\left(\gamma^{\prime} \gamma\right) \sigma\left(\gamma^{\prime}, \gamma\right)
$$

These satisfy

$$
L_{\gamma}^{\sigma} L_{\gamma^{\prime}}^{\sigma}=\sigma\left(\gamma, \gamma^{\prime}\right) L_{\gamma \gamma^{\prime}}^{\sigma} \quad R_{\gamma}^{\sigma} R_{\gamma^{\prime}}^{\sigma}=\sigma\left(\gamma, \gamma^{\prime}\right) R_{\gamma \gamma^{\prime}}^{\sigma} .
$$

The cocycle identity can be used to show that the left $\sigma$-regular representation commutes with the right $\bar{\sigma}$-regular representation, where $\bar{\sigma}$ denotes the conjugate cocycle. Also the left $\bar{\sigma}$-regular representation commutes with the right $\sigma$-regular representation.

Let $\left\{\gamma_{i}\right\}_{i=1}^{r}$ be a symmetric set of generators of $\Gamma$. The Harper operator is given by

$$
\mathcal{R}_{\sigma}=\sum_{i=1}^{r} R_{\gamma_{i}}^{\sigma}
$$




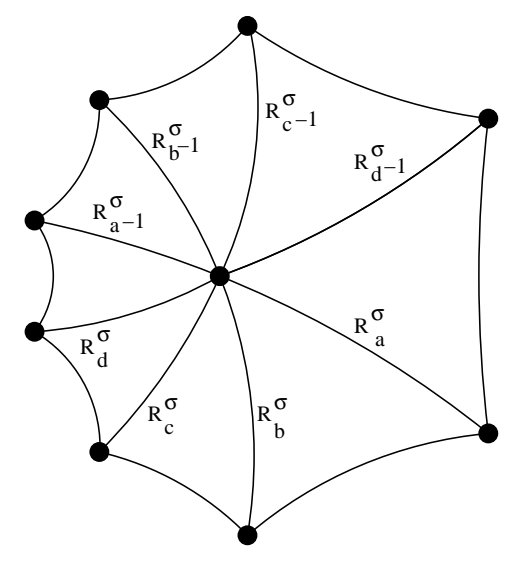

FiguRE 8. Harper operator on a lattice in the hyperbolic plane

The operator $r-\mathcal{R}_{\sigma}$ is the discrete analog of the magnetic Laplacian ( $c f .[\mathbf{3 0}$ ).

Algebra of observables (discrete model). We continue in the same generality as above. The special case of interest for the integer quantum Hall effect will be for $\Gamma=\mathbb{Z}^{2}$, but we adopt a more general setting in view of applications to the fractional case.

For $\Gamma$ a finitely generated discrete group, let $\mathbb{C}(\Gamma, \sigma)$ be the algebra generated by the magnetic translations represented as operators in $\mathcal{B}\left(\ell^{2}(\Gamma)\right)$ through the right $\sigma$-regular representation $R_{\gamma}^{\sigma}$. Equivalently, the algebra $\mathbb{C}(\Gamma, \sigma)$ consists of functions

$$
f: \Gamma \rightarrow \mathbb{C}
$$

with the convolution product

$$
f_{1} * f_{2}(\gamma)=\sum_{\gamma_{1} \gamma_{2}=\gamma} f_{1}\left(\gamma_{1}\right) f_{2}\left(\gamma_{2}\right) \sigma\left(\gamma_{1}, \gamma_{2}\right),
$$

acting on the Hilbert space $\ell^{2}(\Gamma)$.

By taking the weak closure of $\mathbb{C}(\Gamma, \sigma)$ one obtains the twisted group von Neumann algebra $\mathcal{U}(\Gamma, \sigma)$. This is equivalently (by the commutant theorem of von Neumann) described as

$$
\mathcal{U}(\Gamma, \sigma)=\left\{A \in B\left(\ell^{2}(\Gamma)\right):\left[L_{\gamma}^{\bar{\sigma}}, A\right]=0 \forall \gamma \in \Gamma\right\} .
$$

That is, $\mathcal{U}(\Gamma, \sigma)$ is the commutant of the left $\bar{\sigma}$-regular representation. When taking the norm closure of $\mathbb{C}(\Gamma, \sigma)$ one obtains the twisted (reduced) group $C^{*}$-algebra $C_{r}^{*}(\Gamma, \sigma)$, which is the algebra of observables in the discrete model.

The key properties of these algebras are summarized as follows. $\mathcal{U}(\Gamma, \sigma)$ is generated by its projections and it is also closed under the measurable functional calculus, i.e. if $a \in \mathcal{U}(\Gamma, \sigma)$ and $a=a^{*}, a>0$, then $f(a) \in \mathcal{U}(\Gamma, \sigma)$ for all essentially bounded measurable functions $f$ on $\mathbb{R}$. On the other hand, $C_{r}^{*}(\Gamma, \sigma)$ has only at most countably many projections and is only closed under the continuous functional calculus.

In the case when $\sigma=1$ (integer flux), with the group $\Gamma=\mathbb{Z}^{2}$, we simply have $\mathcal{U}(\Gamma, 1) \cong L^{\infty}\left(T^{2}\right)$ and $C^{*}(\Gamma, 1) \cong C\left(T^{2}\right)$, i.e. functions on the classical Brillouin 
zone. Here the ordinary torus $T^{2}$ is identified with the group $\widehat{\Gamma}$ of characters of the abelian group $\Gamma=\mathbb{Z}^{2}$.

In Bellissard's model of the integer quantum Hall effect, where $\Gamma=\mathbb{Z}^{2}$, with $\sigma$ the nontrivial cocycle described in (3.17) and $\theta=\alpha_{2}-\alpha_{1}$, the twisted (reduced) group $C^{*}$-algebra is the irrational rotation algebra of the noncommutative torus,

$$
C_{r}^{*}(\Gamma, \sigma) \cong A_{\theta} .
$$

We will not describe in detail the derivation of the quantization of the Hall conductance in this model of the integer quantum Hall effect. In fact, we will concentrate mostly on a model for the fractional quantum Hall effect and we will show how to recover the integer quantization within that model, using the results of [5].

Spectral theory. For $\Gamma$ a finitely generated discrete group and $\left\{g_{i}\right\}_{i=1}^{r}$ a symmetric set of generators, the Cayley graph $\mathcal{G}=\mathcal{G}\left(\Gamma, g_{i}\right)$ has as set of vertices the elements of $\Gamma$ and as set of edges emanating from a given vertex $h \in \Gamma$ the set of translates $g_{i} h$.

The random walk operator (1.15) for $\Gamma$ is then an average on nearest neighbors in the Cayley graph. The discrete analog of the Schrödinger equation is of the form

$$
i \frac{\partial}{\partial t} \psi=\mathcal{R}_{\sigma} \psi+V \psi
$$

where all physical constants have been set equal to 1 . It describes the quantum mechanics of a single electron confined to move along the Cayley graph of $\Gamma$, subject to the periodic magnetic field. Here $\mathcal{R}_{\sigma}$ is the Harper operator encoding the magnetic field and $V$ is the electric potential of the independent electron approximation. The latter can be taken to be an operator in the twisted group algebra, $V \in \mathbb{C}(\Gamma, \sigma)$. More precisely, equation (3.24) should be formulated with the discrete magnetic laplacian $\delta_{\sigma}=r-\mathcal{R}_{\sigma}$ in place of $\mathcal{R}_{\sigma}$, with $r$ the cardinality of a symmetric set of generators for $\Gamma$. This does not really matter as far as the spectral properties are concerned, as the spectrum of one determines the spectrum of the other.

As in the case of the theory of electrons in solids without magnetic field recalled in the first section, an important problem is understanding the energy levels of the Hamiltonian $H_{\sigma, V}=\mathcal{R}_{\sigma}+V$, and the band structure (gaps in the spectrum).

The Harper operator $\mathcal{R}_{\sigma}$ is a bounded self-adjoint operator on $\ell^{2}(\Gamma)$, since it is defined in terms of a symmetric set of generators of $\Gamma$. Thus, the $\operatorname{spectrum~} \operatorname{Spec}\left(\mathcal{R}_{\sigma}\right)$ is a closed and bounded subset of $\mathbb{R}$. It follows that the complement $\mathbb{R} \backslash \operatorname{Spec}\left(\mathcal{R}_{\sigma}\right)$ is an open subset of $\mathbb{R}$, hence a countable union of disjoint open intervals. Each such interval is called a gap in the spectrum.

There are two very different situations. When the complement of the spectrum consists of a finite collection of intervals then the operator has a band structure, while if the complement consists of an infinite collection of intervals then the spectrum is a Cantor set. In the case of the group $\Gamma=\mathbb{Z}^{2}$, one or the other possibility occurs depending on the rationality or irrationality of the flux

$$
\theta=\langle[\sigma],[\Gamma]\rangle .
$$

This gives rise to a diagram known as the Hofstadter butterfly (Figure 9). 


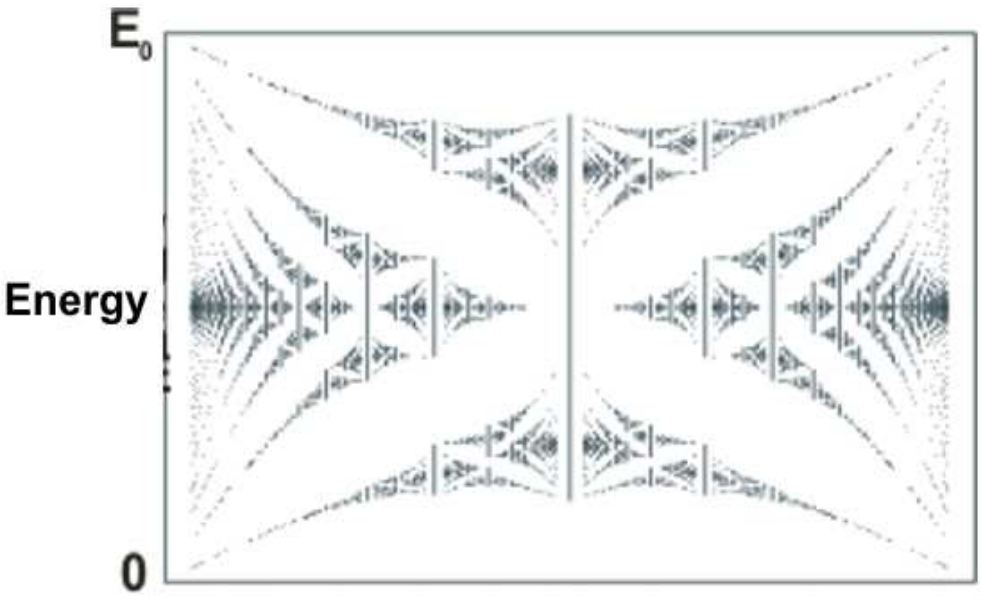

Magnetic Flux $\Phi / \Phi_{0}$

FiguRE 9. Hofstadter butterfly

Range of the trace. In our model of the fractional quantum Hall effect, $\Gamma$ is a cocompact Fuchsian group of signature $\left(g, \nu_{1}, \ldots, \nu_{n}\right)$. In this case $(c f$. [25]), if $[\sigma]$ is rational, then there is only a finite number of gaps in the spectrum of $H_{\sigma}+V$. In fact, if $\theta=p / q$ then the number of gaps is at most

$$
(q+1) \prod_{j=1}^{n}\left(\nu_{j}+1\right)
$$

In terms of the algebra of observables, the question of how many gaps there are in the spectrum of $H_{\sigma, V}$ can be reduced to studying the number of projections in the $C^{*}$-algebra $C_{r}^{*}(\Gamma, \sigma)$ (up to equivalence). In fact, we have

$$
H_{\sigma, V} \in \mathbb{C}(\Gamma, \sigma) \subset C_{r}^{*}(\Gamma, \sigma) \subset \mathcal{U}(\Gamma, \sigma) .
$$

In particular, $H_{\sigma}$ and its spectral projections

$$
P_{E}=\chi_{(-\infty, E]}\left(H_{\sigma, V}\right)
$$

belong to the algebra $\mathcal{U}(\Gamma, \sigma)$. Moreover, when $E \notin \operatorname{Spec}\left(H_{\sigma, V}\right)$, the spectral projection $P_{E}$ is in $C_{r}^{*}(\Gamma, \sigma)$. In fact, suppose that the spectrum of $H_{\sigma, V}$ is contained in a closed interval, and that the open interval $(a, b)$ is a spectral gap of $H_{\sigma, V}$. Suppose that $E \in(a, b)$, i.e. $E \notin \operatorname{Spec}\left(H_{\sigma, V}\right)$. Then there is a holomorphic function $\phi$ on a neighborhood of $\operatorname{spec}\left(H_{\sigma, V}\right)$ such that

$$
P_{E}=\phi\left(H_{\sigma, V}\right)=\int_{C} \frac{d \lambda}{\lambda-H_{\sigma, V}}
$$

where $C$ is a closed contour enclosing the spectrum of $H_{\sigma, V}$ to the left of $E$. Since $C_{r}^{*}(\Gamma, \sigma)$ is closed under the holomorphic functional calculus, it follows that $P_{E} \in$ $C_{r}^{*}(\Gamma, \sigma)$.

The equivalence relation we need to consider on projections, so that the counting will provide the counting of spectral gaps, is described as follows. Let $\operatorname{Proj}\left(C_{r}^{*}(\Gamma, \sigma) \otimes\right.$ $\mathcal{K})$ denote the projections in $C_{r}^{*}(\Gamma, \sigma) \otimes \mathcal{K}$, where $\mathcal{K}$ the $C^{*}$ algebra of compact 
operators. Two projections $P, Q \in \operatorname{Proj}\left(C_{r}^{*}(\Gamma, \sigma) \otimes \mathcal{K}\right)$ are said to be Murray-von Neumann equivalent if there is an element $V \in C_{r}^{*}(\Gamma, \sigma) \otimes \mathcal{K}$ such that $P=V^{*} V$ and $Q=V V^{*}$, and we write $P \sim Q$. It can be shown that $\operatorname{Proj}\left(C_{r}^{*}(\Gamma, \sigma) \otimes \mathcal{K}\right) / \sim$ is an abelian semi-group under direct sums, and the Grothendieck group $\left.K_{0}\left(C_{r}^{*}(\Gamma, \sigma)\right)\right)$ is defined as the associated abelian group.

Now the estimate on the number of equivalence classes of projections is achieved by computing the range of a trace. The von Neumann algebra $\mathcal{U}(\Gamma, \sigma)$ and $C^{*}$-algebra $C_{r}^{*}(\Gamma, \sigma)$ have a canonical faithful finite trace $\tau$, where

$$
\tau(a)=\left\langle a \delta_{1}, \delta_{1}\right\rangle_{\ell^{2}(\Gamma)},
$$

where $\delta_{\gamma}$ is the basis of $\ell^{2}(\Gamma)$. If $\operatorname{Tr}$ denotes the standard trace on bounded operators in an $\infty$-dimensional separable Hilbert space $\mathcal{H}$, then we obtain a trace

$$
\operatorname{tr}=\tau \otimes \operatorname{Tr}: \operatorname{Proj}\left(C_{r}^{*}(\Gamma, \sigma) \otimes \mathcal{K}\right) \rightarrow \mathbb{R}
$$

This induces a trace on the $K$-group

$$
\left.[\operatorname{tr}]: K_{0}\left(C_{r}^{*}(\Gamma, \sigma)\right)\right) \rightarrow \mathbb{R}
$$

with

$$
\operatorname{tr}\left(\operatorname{Proj}\left(C_{r}^{*}(\Gamma, \sigma)\right)\right)=[\operatorname{tr}]\left(K_{0}\left(C_{r}^{*}(\Gamma, \sigma)\right)\right) \cap[0,1] .
$$

The result quoted above in (3.25), counting the energy gaps in our hyperbolic model, can then be derived from the following result proved in [25.

THEOREM 3.1. Let $\Gamma$ be a cocompact Fuchsian group of signature $\left(g: \nu_{1}, \ldots, \nu_{n}\right)$ and $\sigma$ be a multiplier on $\Gamma$ with flux $\theta$. Then the range of the trace is,

$$
[\operatorname{tr}]\left(K_{0}\left(C_{r}^{*}(\Gamma, \sigma)\right)\right)=\mathbb{Z}+\theta \mathbb{Z}+\sum_{j} \frac{1}{\nu_{j}} \mathbb{Z} .
$$

Here the flux is again given by the pairing $\theta=\langle[\sigma],[\Gamma]\rangle$, where $[\Gamma]=\frac{\left[\Sigma_{g^{\prime}}\right]}{\# G}$ is the fundamental class of the group $\Gamma=\Gamma\left(g ; \nu_{1}, \ldots, \nu_{n}\right)$ and $g^{\prime}$ is given by the formula (3.5).

The Baum-Connes conjecture holds for the Fuchsian groups $\Gamma=\Gamma\left(g ; \nu_{1}, \ldots, \nu_{n}\right)$, and one can compute the $K$-theory of the $C^{*}$-algebra $C_{r}^{*}(\Gamma, \sigma)$ in terms of the orbifold K-theory of $\Sigma\left(g ; \nu_{1}, \ldots, \nu_{n}\right)$. This uses a Morita equivalence $\left(A \otimes C_{0}(G)\right) \rtimes$ $\Gamma \simeq C_{0}(\Gamma \backslash G, \mathcal{E})$, where $\mathcal{E}=A \times_{\Gamma} G \rightarrow \Gamma \backslash G$, in the case where $G=\operatorname{PSL}(2, \mathbb{R})$. Without the twisting by $\sigma$, one can identify

$$
K_{\bullet}\left(C_{r}^{*}(\Gamma)\right) \cong K_{S O(2)}^{\bullet}\left(P\left(g ; \nu_{1}, \ldots, \nu_{n}\right)\right),
$$

where $P\left(g ; \nu_{1}, \ldots, \nu_{n}\right)$ is the frame bundle $\Gamma \backslash \operatorname{PSL}(2, \mathbb{R})$. The result can be identified with the orbifold K-theory

$$
K_{\text {orb }}^{\bullet}\left(\Sigma\left(g ; \nu_{1}, \ldots, \nu_{n}\right)\right) \cong \begin{cases}\mathbb{Z}^{2-n+\sum \nu_{j}} & \bullet=\text { even } \\ \mathbb{Z}^{2 g} & \bullet=\text { odd }\end{cases}
$$

In the twisted case, one still has the equivalence $C_{0}(\Gamma \backslash G, \mathcal{E}) \simeq C_{0}\left(\Gamma \backslash G, \mathcal{E}_{\sigma}\right)$ when the class $\delta(\sigma)=0$, where $\delta: H^{2}(\Gamma, U(1)) \rightarrow H^{3}(\Gamma, \mathbb{Z})$ is a surjection coming from the long exact sequence of $1 \rightarrow \mathbb{Z} \hookrightarrow \mathbb{R} \stackrel{\exp (2 \pi i \cdot)}{\longrightarrow} U(1) \rightarrow 1$.

The computation of the range of the trace (3.27) then follows from an index theorem. Let $\mathcal{E}$ be an orbifold vector bundle over $\Sigma=\Sigma\left(g ; \nu_{1}, \ldots, \nu_{n}\right)$, and $[\mathcal{E}] \in K_{\text {orb }}^{\bullet}(\Sigma)$. Let $\tilde{\phi}_{\mathcal{E}}^{+}$be the twisted Dirac operator on the universal cover $\mathbb{H}$. 
For $\nabla^{2}=i \omega$ the magnetic field, the operator $\tilde{\phi}_{\mathcal{E}}^{+} \otimes \nabla$ commutes with the projective action of $(\Gamma, \sigma)$. There is an analytic index

$$
\operatorname{ind}_{(\Gamma, \sigma)} \tilde{\phi}_{\mathcal{E}}^{+} \otimes \nabla \in K_{0}\left(C_{r}^{*}(\Gamma, \sigma)\right),
$$

which is the image under the (twisted) Kasparov map

$$
\mu_{\sigma}([\mathcal{E}])=\operatorname{ind}_{(\Gamma, \sigma)} \tilde{\phi}_{\mathcal{E}}^{+} \otimes \nabla .
$$

To compute the range of the trace $[\operatorname{tr}]: K_{0}\left(C_{r}^{*}(\Gamma, \sigma)\right) \rightarrow \mathbb{R}$ one computes then the index

$$
\operatorname{Ind}_{L^{2}}\left(\tilde{\partial}_{\mathcal{E}}^{+} \otimes \nabla\right)=[\operatorname{tr}]\left(\operatorname{ind}_{(\Gamma, \sigma)} \tilde{\partial}_{\mathcal{E}}^{+} \otimes \nabla\right) .
$$

We have

$$
\operatorname{Ind}_{L^{2}}\left(\tilde{\partial}_{\mathcal{E}}^{+} \otimes \nabla\right)=\frac{1}{2 \pi} \int_{\Sigma} \hat{A} \operatorname{tr}\left(e^{R^{\mathcal{E}}}\right) e^{\omega}
$$

Since $\Sigma$ is of real dimension 2, this formula reduces to

$$
\frac{\operatorname{rank\mathcal {E}}}{2 \pi} \int_{\Sigma} \omega+\frac{1}{2 \pi} \int_{\Sigma} \operatorname{tr}\left(R^{\mathcal{E}}\right)
$$

The first term is computed by

$$
\frac{\operatorname{rank}(\mathcal{E})}{2 \pi} \int_{\Sigma} \omega=\frac{\operatorname{rank}(\mathcal{E})}{2 \pi \# G} \int_{\Sigma_{g^{\prime}}} \omega=\operatorname{rank}(\mathcal{E})\langle[\sigma],[\Gamma]\rangle \in \theta \mathbb{Z}
$$

while the second term is computed by the Kawasaki index theorem for orbifolds

$$
\mathbb{Z} \ni \operatorname{ind}\left(\not \not_{\mathcal{E}}^{+}\right)=\frac{1}{2 \pi} \int_{\Sigma} \operatorname{tr}\left(R^{\mathcal{E}}\right)+\frac{1}{2 \pi} \sum_{i=1}^{n} \frac{\beta_{i}}{\nu_{i}}
$$

where $\left(\beta_{i}, \nu_{i}\right)$ are the Seifert invariants of $\mathcal{E}$. This implies that

$$
\frac{1}{2 \pi} \int_{\Sigma} \operatorname{tr}\left(R^{\mathcal{E}}\right) \in \mathbb{Z}+\sum_{i=1}^{n} \frac{1}{\nu_{i}} \mathbb{Z}
$$

Rieffel, and Pimsner and Voiculescu established analogous results in the case $\Gamma=$ $\mathbb{Z}^{2}$. The result in the case of torsion-free Fuchsian groups was established in $\mathbf{5}$. In more recent work, Mathai generalized this result to discrete subgroups of rank 1 groups and to all amenable groups, and more generally whenever the BaumConnes conjecture with coefficients holds for the discrete group, 26. By contrast, the behavior of spectral gaps when the flux is irrational is still mysterious. The problem can be formulated in terms of the following conjecture (also known as the "generalized ten Martini problem"), cf. 24] 25].

ConjeCture 3.1. Let $\Gamma$ be a cocompact Fuchsian group and $\sigma$ be a multiplier on $\Gamma$. If the flux $\theta$ is irrational, then there is a $V \in \mathbb{C}(\Gamma, \sigma)$ such that $H_{\sigma, V}$ has an infinite number of gaps in its spectrum.

It is not yet known if any gaps exist at all in this case! However, using Morse-type potentials, Mathai and Shubin $\mathbf{2 7}$ proved that there is an arbitrarily large number of gaps in the spectrum of magnetic Schrödinger operators on covering spaces, (i.e. in the continuous model).

Recent work of Dodziuk, Mathai, and Yates 14 shows another interesting property of the spectrum, namely the fact that all $L^{2}$ eigenvalues of the Harper operators 
of surface groups $\Gamma$ are algebraic numbers, whenever the multiplier is algebraic, that is, when $[\sigma] \in H^{2}(\Gamma, \overline{\mathbb{Q}} / \mathbb{Z})$. In fact the same result remains true when adding potentials $V$ in $\overline{\mathbb{Q}}(\Gamma, \sigma)$ to the Harper operator.

\section{Hall conductance}

We finally come to a discussion of the quantization of the Hall conductance. This will follow again from a topological argument, and index theorem, as in the Bellissard case, but in our setting with hyperbolic geometry. We will derive, from our model, a formula for the Hall conductance in terms of values of the orbifold Euler characteristic, and we will compare the results with experimentally observed values.

A smooth subalgebra. We will consider a cyclic cocycle associated to the Connes-Kubo formula for the conductance, which will be defined in terms of certain derivations. For this reason, we need to introduce a smooth subalgebra, namely, a dense involutive subalgebra of the algebra of observables $C_{r}^{*}(\Gamma, \sigma)$. This subalgebra contains $\mathbb{C}(\Gamma, \sigma)$ and is contained in the domain of definition of the derivations. It contains the spectral projection $P_{E}$, when the Fermi level is in a gap of the energy spectrum. Moreover, it satisfies the following two key properties.

(1) The inclusion $\mathcal{R} \subset C_{r}^{*}(\Gamma, \sigma)$ induces an isomorphism in $K$-theory.

(2) Polynomial growth group cocycles on $\Gamma$ define cyclic cocycles on $\mathbb{C}(\Gamma, \sigma)$ that extend continuously to $\mathcal{R}$.

$\mathcal{R}$ is defined as follows. Consider an operator $D$ defined as

$$
D \delta_{\gamma}=\ell(\gamma) \delta_{\gamma} \forall \gamma \in \Gamma
$$

where $\ell(\gamma)$ denotes the word length of $\gamma$. Let $\delta=\operatorname{ad}(D)$ denote the commutator $[D, \cdot]$. Then $\delta$ is an unbounded, but closed derivation on $C_{r}^{*}(\Gamma, \sigma)$. Define

$$
\mathcal{R}:=\bigcap_{k \in \mathbb{N}} \operatorname{Dom}\left(\delta^{k}\right) .
$$

It is clear that $\mathcal{R}$ contains $\delta_{\gamma} \forall \gamma \in \Gamma$ and so it contains $\mathbb{C}(\Gamma, \sigma)$. Hence it is dense in $C_{r}^{*}(\Gamma, \sigma)$. It is not hard to see that $\mathcal{R}$ is closed under the holomorphic functional calculus, and therefore by a result of Connes, property (1) above holds, and by equation (3.26), $P_{E} \in \mathcal{R}$.

Until now, we have not used any special property of the group $\Gamma$. But now assume that $\Gamma$ is a surface group. Then it follows from a result of $\mathbf{2 0}, \mathbf{2 2}$ that there is a $k \in \mathbb{N}$ and a positive constant $C^{\prime}$ such that for all $f \in \mathbb{C}(\Gamma, \sigma)$, one has the Haagerup inequality

$$
\|f\| \leq C^{\prime} \nu_{k}(f)
$$

where $\|f\|$ denotes the operator norm of the operator on $\ell^{2}(\Gamma)$ given by left convolution by $f$, and the bound $\nu_{k}(f)$ is given in terms of the $L^{2}$ norms of $f$ and of $\left(1+l^{2}\right)^{s / 2} f$, for all $0 \leq s \leq k$. Using this, it is routine to show that property (2) holds.

Notice that the spectral projections onto gaps in the Hamiltonian $H$ belong to the algebra of observables $\mathcal{R}$, for any choice of electric potential $V$. 
Cyclic cocycles. Cyclic cohomology was introduced by Connes in 11. It is a main source of invariants of noncommutative spaces, obtained by the pairing of cyclic cocycles with $K$-theory. Cyclic cocycles are also called multilinear traces, and the word cyclic refers to invariance under the cyclic group $\mathbb{Z} /(n+1) \mathbb{Z}$ acting on the slots of the Cartesian product. Namely, $t$ is a cyclic $n$-cocycle if

$$
t: \mathcal{R} \times \mathcal{R} \cdots \times \mathcal{R} \rightarrow \mathbb{C}
$$

satisfies the cyclic condition

$$
t\left(a_{0}, a_{1}, \ldots, a_{n}\right)=t\left(a_{n}, a_{0}, a_{1}, \ldots, a_{n-1}\right)=\cdots=t\left(a_{1}, \ldots, a_{n}, a_{0}\right),
$$

and the cocycle condition

$$
t\left(a a_{0}, a_{1}, \ldots, a_{n}\right)-t\left(a, a_{0} a_{1}, \ldots, a_{n}\right) \cdots(-1)^{n+1} t\left(a_{n} a, a_{0}, \ldots, a_{n-1}\right)=0 .
$$

For instance, a cyclic 0-cocycle is just a trace. In fact, in this case, the condition it satisfies is $t(a b)=t(b a)$. A cyclic 1-cocycle satisfies $t(a, b)=t(b, a)$ and $t(a b, c)-$ $t(a, b c)+t(c a, b)=0$, and a cyclic 2-cocycle satisfies

$$
\begin{gathered}
t(a, b, c)=t(c, a, b)=t(b, c, a) \quad \text { and } \\
t(a b, c, d)-t(a, b c, d)+t(a, b, c d)-t(d a, b, c)=0 .
\end{gathered}
$$

Conductance cocycle. A formula for the Hall conductance is obtained from transport theory. In the case of $\Gamma=\mathbb{Z}^{2}$, the current density in $e_{1}$ direction corresponds to the functional derivative $\delta_{1}$ of $H_{\sigma}$ by $A_{1}$, the corresponding component of the magnetic potential. The expected value of current is the given by $\operatorname{tr}\left(P \delta_{1} H\right)$ for a state $P$ of the system. Using $\partial_{t} P=i[P, H]$ and $\partial_{t}=\frac{\partial A_{2}}{\partial t} \times \delta_{2}$, where $e_{2} \perp e_{1}$, one gets

$$
i \operatorname{tr}\left(P\left[\partial_{t} P, \delta_{1} P\right]\right)=-i E_{2} \operatorname{tr}\left(P\left[\delta_{2} P, \delta_{1} P\right]\right),
$$

where the electrostatic potential has been gauged away, leaving $\mathbf{E}=-\frac{\partial \mathbf{A}}{\partial t}$. Because the charge carriers are Fermions, two different charge carriers must occupy different quantum eigenstates of the Hamiltonian $H$. In the zero temperature limit, charge carriers occupy all levels below the Fermi level, so that we can set $P=P_{F}$ in the formula above. This gives the Kubo formula for the conductance

$$
\sigma_{H}=\operatorname{tr}\left(P_{F}\left[\delta_{1} P_{F}, \delta_{2} P_{F}\right]\right) .
$$

This argument can be generalized to our setting, keeping into account the fact that, in our model, by effect of the strong multi-electron interaction, to a moving elector the directions $\left\{e_{1}, e_{2}\right\}$ appear split into $\left\{e_{i}, e_{i+g}\right\}_{i=1, \ldots, g}$ corresponding to $a_{i}, b_{i}$, for some lattice in the hyperbolic plane. The following is a general mathematical formulation of the result.

Given a 1-cocycle $a$ on the discrete group $\Gamma$, i.e.

$$
a\left(\gamma_{1} \gamma_{2}\right)=a\left(\gamma_{1}\right)+a\left(\gamma_{2}\right) \quad \forall \gamma_{1}, \gamma_{2} \in \Gamma,
$$

one can define a linear functional $\delta_{a}$ on the twisted group algebra $\mathbb{C}(\Gamma, \sigma)$

$$
\delta_{a}(f)(\gamma)=a(\gamma) f(\gamma)
$$


Then one verifies that $\delta_{a}$ is a derivation:

$$
\begin{aligned}
& \delta_{a}(f g)(\gamma)=a(\gamma) f g(\gamma) \\
& =a(\gamma) \sum_{\gamma=\gamma_{1} \gamma_{2}} f\left(\gamma_{1}\right) g\left(\gamma_{2}\right) \sigma\left(\gamma_{1}, \gamma_{2}\right) \\
& =\sum_{\gamma=\gamma_{1} \gamma_{2}}\left(a\left(\gamma_{1}\right)+a\left(\gamma_{2}\right)\right) f\left(\gamma_{1}\right) g\left(\gamma_{2}\right) \sigma\left(\gamma_{1}, \gamma_{2}\right) \\
& =\sum_{\gamma=\gamma_{1} \gamma_{2}}\left(\delta_{a}(f)\left(\gamma_{1}\right) g\left(\gamma_{2}\right) \sigma\left(\gamma_{1}, \gamma_{2}\right)+f\left(\gamma_{1}\right) \delta_{a}(g)\left(\gamma_{2}\right) \sigma\left(\gamma_{1}, \gamma_{2}\right)\right) \\
& =\left(\delta_{a}(f) g\right)(\gamma)+\left(f \delta_{a} g\right)(\gamma) .
\end{aligned}
$$

In the case of a Fuchsian group $\Gamma$, the first cohomology $H^{1}(\Gamma, \mathbb{Z})$ of the group $\Gamma$ is a free Abelian group of rank $2 g$, where $g$ is the genus of $\Gamma \backslash \mathbb{H}$. The cohomology $H^{1}(\Gamma, \mathbb{R})$ is in fact a symplectic vector space, and we can assume that $\left\{a_{j}, b_{j}\right\}_{j=1, \ldots, g}$ is a symplectic basis.

We denote $\delta_{a_{j}}$ by $\delta_{j}$ and $\delta_{b_{j}}$ by $\delta_{j+g}$. These derivations give rise to a cyclic 2-cocycle on the twisted group algebra $\mathbb{C}(\Gamma, \sigma)$,

$$
\operatorname{tr}_{K}\left(f_{0}, f_{1}, f_{2}\right)=\sum_{j=1}^{g} \operatorname{tr}\left(f_{0}\left(\delta_{j}\left(f_{1}\right) \delta_{j+g}\left(f_{2}\right)-\delta_{j+g}\left(f_{1}\right) \delta_{j}\left(f_{2}\right)\right)\right) .
$$

$\operatorname{tr}_{K}$ is called the conductance 2-cocycle.

Let $P_{E}$ denote denote the spectral projection associated to the Fermi level, i.e. $P_{E}=\chi_{(-\infty, E]}(H)$. Then, in the zero temperature limit, the Hall conductance is given by

$$
\sigma_{E}=\operatorname{tr}_{K}\left(P_{E}, P_{E}, P_{E}\right)
$$

Quantum adiabatic limit. We recall briefly the justification of (4.2) in terms of the quantum adiabatic limit for a slowly varying time dependent Hamiltonian, cf. 7 .

If $H(s)$ is a smooth family of self-adjoint Hamiltonians and $P(s)$ are spectral projections on a gap in the spectrum of $H(s)$, then

$$
X(s)=\frac{1}{2 \pi i} \oint_{C} R(z, s) \partial_{s} P(s) R(z, s) d z,
$$

with $R(z, s)=(H(s)-z)^{-1}$, satisfies the commutation relations

$$
\left[\partial_{s} P(s), P(s)\right]=[H(s), X(s)] .
$$

The quantum adiabatic limit theorem ( $c f$. [2] ) then shows that the adiabatic evolution approximates well the physical evolution, for large values of the adiabatic parameter $\tau \rightarrow \infty$, via an estimate of the form

$$
\begin{gathered}
\left\|\left(U_{\tau}(s)-U_{a}(s)\right) P(0)\right\| \leq \\
\frac{1}{\tau} \max _{s \in[0, \infty)}\left\{2\|X(s) P(s)\|+\left\|\partial_{s}(X(s) P(s)) P(s)\right\|\right\} .
\end{gathered}
$$

Here the physical evolution satisfies

$$
i \partial_{s} U_{\tau}(s)=\tau H(s) U_{\tau}(s),
$$

$U_{\tau}(0)=1$, where $s=t / \tau$ is a scaled time, and the adiabatic evolution is defined by the equation

$$
P(s)=U_{a}(s) P(0) U_{a}(s)^{*}
$$


with $U_{a}(0)=1$.

In our setting, the functional derivative $\delta_{k} H$, with respect to a component $A_{k}$ of the magnetic potential, gives a current density $J_{k}$. Its expectation value in a state described by a projection $P$ on a gap in the spectrum of the Hamiltonian is then computed by $\operatorname{tr}\left(P \delta_{k} H\right)$. In the quantum adiabatic limit, one can replace $\delta_{k} H$ with $\delta_{k} H_{a}$, where the adiabatic Hamiltonian $H_{a}$ satisfies

$$
i \partial_{s} U_{a}(s)=\tau H_{a}(s) U_{a}(s)
$$

and the equation of motion

$$
\left[H_{a}(s), P(s)\right]=\frac{i}{\tau} \partial_{s} P(s) .
$$

This implies that the relation

$$
\operatorname{tr}\left(P\left[\partial_{t} P, \delta_{k} P\right]\right)=i \operatorname{tr}\left(\delta_{k}\left(P H_{a}\right)\right)-i \operatorname{tr}\left(P \delta_{k} H_{a}\right) .
$$

We make some simplifying assumptions. If the trace is invariant under variations of $A_{k}$, then the first term in the right hand side of (4.3) vanishes. We also assume that the only time dependence of $H$ and $P$ is in the adiabatic variation of a component $A_{j}$ distinct from $A_{k}$, and we work in the Landau gauge, so that the electrostatic potential vanishes and the electric field is given by $\mathbf{E}=-\partial \mathbf{A} / \partial t$. Then we have $\partial_{t}=-E_{j} \delta_{j}$, so that the expectation of the current $J_{k}$ is given by

$$
\begin{gathered}
\operatorname{tr}\left(P \delta_{k} H\right)=i \operatorname{tr}\left(P\left[\partial_{t} P, \delta_{k} P\right]\right) \\
\quad=-i E_{j} \operatorname{tr}\left(P\left[\delta_{j} P, \delta_{k} P\right]\right),
\end{gathered}
$$

hence the conductance for a current in the $k$ direction induced by an electric field in the $j$ direction is given by $-i \operatorname{tr}\left(P\left[\delta_{j} P, \delta_{k} P\right]\right)$. The analytic aspects of this formal argument can be made rigorous following the techniques used in $\mathbf{3 3}$.

Area cocycle. Our conclusion above, as in the case of the integer Hall effect, is that one can compute the Hall conductance by evaluating a certain cyclic cocycle on a projection, namely on some element in K-theory. It is often the case that, in order to compute the pairing of a cyclic cocycle with K-theory, one can simplify the problem by passing to another cocycle in the same cohomology class, i.e. that differs by a coboundary. This is what will happen in our case.

We introduce another cyclic cocycle, which has a more direct geometric meaning. On $G=\operatorname{PSL}(2, \mathbb{R})$, there is an area cocycle $(c f$. 12 $)$. This is the 2-cocycle

$$
\begin{aligned}
C: G \times G \rightarrow & \mathbb{R} \\
C\left(\gamma_{1}, \gamma_{2}\right)= & \text { (oriented) hyperbolic area of the } \\
& \text { geodesic triangle with } \\
& \text { vertices at }\left(z_{0}, \gamma_{1}^{-1} z_{0}, \gamma_{2} z_{0}\right), \quad z_{0} \in \mathbb{H}
\end{aligned}
$$

The restriction of this cocycle to a discrete subgroup $\Gamma \subset \operatorname{PSL}(2, \mathbb{R})$ gives the area group cocycle on $\Gamma$. This in turn defines a cyclic 2-cocycle on $\mathbb{C}(\Gamma, \sigma)$ by

$$
\operatorname{tr}_{C}\left(f_{0}, f_{1}, f_{2}\right)=\sum_{\gamma_{0} \gamma_{1} \gamma_{2}=1} f_{0}\left(\gamma_{0}\right) f_{1}\left(\gamma_{1}\right) f_{2}\left(\gamma_{2}\right) C\left(\gamma_{1}, \gamma_{2}\right) \sigma\left(\gamma_{1}, \gamma_{2}\right) .
$$

Since $C$ is (polynomially) bounded, $\operatorname{tr}_{C}$ can be shown to extend to the smooth subalgebra $\mathcal{R}$. 
Comparison. Two cyclic 2-cocycles $t_{1}$ and $t_{2}$ differ by a coboundary (that is, they define the same cyclic cohomology class) iff

$$
t_{1}\left(a_{0}, a_{1}, a_{2}\right)-t_{2}\left(a_{0}, a_{1}, a_{2}\right)=\lambda\left(a_{0} a_{1}, a_{2}\right)-\lambda\left(a_{0}, a_{1} a_{2}\right)+\lambda\left(a_{2} a_{0}, a_{1}\right),
$$

where $\lambda$ is a cyclic 1-cocycle.

As in 5], 24], the difference between the conductance cocycle $\operatorname{tr}_{K}$ and the area cocycle $\operatorname{tr}_{C}$ can be evaluated in terms of the difference between the hyperbolic area of a geodesic triangle and the Euclidean area of its image under the Abel-Jacobi map. This difference can be expressed as a sum of three terms

$$
U\left(\gamma_{1}, \gamma_{2}\right)=h\left(\gamma_{2}^{-1}, 1\right)-h\left(\gamma_{1}^{-1}, \gamma_{2}\right)+h\left(1, \gamma_{1}\right),
$$

where each term is a difference of line integrals, one along a geodesic segment in $\mathbb{H}$ and one along a straight line in the Jacobian variety. The cocycles correspondingly differ by

$$
\operatorname{tr}_{K}\left(f_{0}, f_{1}, f_{2}\right)-\operatorname{tr}_{C}\left(f_{0}, f_{1}, f_{2}\right)=\sum_{\gamma_{0} \gamma_{1} \gamma_{2}=1} f_{0}\left(\gamma_{0}\right) f_{1}\left(\gamma_{1}\right) f_{2}\left(\gamma_{2}\right) U\left(\gamma_{1}, \gamma_{2}\right) \sigma\left(\gamma_{1}, \gamma_{2}\right) .
$$

This expression can be written as $\lambda\left(f_{0} f_{1}, f_{2}\right)-\lambda\left(f_{0}, f_{1} f_{2}\right)+\lambda\left(f_{2} f_{0}, f_{1}\right)$ where

$$
\lambda\left(f_{0}, f_{1}\right)=\sum_{\gamma_{0} \gamma_{1}=1} f_{0}\left(\gamma_{0}\right) f_{1}\left(\gamma_{1}\right) h\left(1, \gamma_{1}\right) \sigma\left(\gamma_{0}, \gamma_{1}\right),
$$

with $h$ as in 4.5).

Thus, the cocycles $\operatorname{tr}_{K}$ and $\operatorname{tr}_{C}$ differ by a coboundary. Since they are cohomologous, $\operatorname{tr}_{K}$ and $\operatorname{tr}_{C}$ induce the same map on $K$-theory.

Values of the Hall conductance. The problem of deriving the values of the Hall conductance is now reduced to computing the pairing of the area cyclic 2cocycle with $K$-theory. The computation is again done through an index theorem. This time the appropriate framework is (a twisted version of) the Connes-Moscovici higher index theorem 13. We have the following result, 24:

THEOREM 4.1. The values of the Hall conductance are given by the twisted higher index formula

$$
\operatorname{Ind}_{c, \Gamma, \sigma}\left(\not \partial_{\mathcal{E}}^{+} \otimes \nabla\right)=\frac{1}{2 \pi \# G} \int_{\Sigma_{g^{\prime}}} \hat{A} \operatorname{tr}\left(e^{R_{\mathcal{E}}}\right) e^{\omega} u_{c},
$$

where $\omega=d \eta$ is the 2-form of the magnetic field, $\nabla^{2}=i \omega, c$ is a cyclic cocycle $c$ and $u_{c}$ is its lift, as in [13, to a 2-form on $\Sigma_{g^{\prime}}$.

Again, since $\Sigma$ is 2-dimensional, the formula (4.6) reduces to just the term

$$
\operatorname{Ind}_{c, \Gamma, \sigma}\left(\not \partial_{\mathcal{E}}^{+} \otimes \nabla\right)=\frac{\operatorname{rank} \mathcal{E}}{2 \pi \# G} \int_{\Sigma_{g^{\prime}}} u_{c} .
$$

Notice that, while it seems at first that in (4.7) all dependence on the magnetic field has disappeared in this formula, in fact it is still present through the orbifold vector bundle $\mathcal{E}$ that corresponds (through Baum-Connes) to the class of the spectral projection $P_{E}$ in $K_{0}\left(C_{r}^{*}(\Gamma, \sigma)\right.$, of the Fermi level. 
When $c$ is the area cocycle, the corresponding 2-form $u_{c}$ is just the hyperbolic volume form, hence the right hand side of (4.7) is computed by the Gauss-Bonnet formula $\int_{\Sigma_{g^{\prime}}} u_{c}=2 \pi\left(2 g^{\prime}-2\right)$, so that

$$
\frac{\operatorname{rank}(\mathcal{E})}{2 \pi \# G} \int_{\Sigma_{g^{\prime}}} u_{c}=\operatorname{rank}(\mathcal{E}) \frac{\left(2 g^{\prime}-2\right)}{\# G}=-\operatorname{rank}(\mathcal{E}) \chi_{o r b}(\Sigma) \in \mathbb{Q}
$$

which yields an integer multiple of the orbifold Euler characteristic.

The conclusion is that, in our model, the Hall conductance takes rational values that are integer multiples of orbifold Euler characteristics, Rational values of the conductance

$$
\sigma_{H}=\operatorname{tr}^{K}\left(P_{F}, P_{F}, P_{F}\right)=\operatorname{tr}^{C}\left(P_{F}, P_{F}, P_{F}\right) \in \mathbb{Z} \chi_{\text {orb }}(\Sigma) .
$$

Discussion of the model. A first important observation, in terms of physical predictions, is that our model of FQHE predicts the existence of an absolute lower bound on the fractional values of the Hall conductance. The lower bound is imposed by the orbifold geometry, and does not have an analog in other theoretical models, hence it appears to be an excellent possible experimental test of the validity of our theoretical model. The lower bound is obtained from the Hurwitz theorem, which states that the maximal order of a finite group $G$ acting by isometries on a smooth Riemann surface $\Sigma_{g^{\prime}}$ is $\# G=84\left(g^{\prime}-1\right)$. This imposes the constraint on the possible quantum Hall fractions:

$$
\phi \geq \frac{2\left(g^{\prime}-1\right)}{84\left(g^{\prime}-1\right)}=\frac{1}{42} .
$$

The lower bound is realized by $1 / 42=-\chi_{\text {orb }}(\Sigma(0 ; 2,3,7))$.

A key advantage of our hyperbolic model is that it treats the FQHE within the same framework developed by Bellissard et al. for the IQHE, with hyperbolic geometry replacing Euclidean geometry, to account for the effect of electron correlation, while remaining formally within a single particle model.

The fractions for the Hall conductance that we get are obtained from an equivariant index theorem and are thus topological in nature. Consequently, the Hall conductance is seen to be stable under small deformations of the Hamiltonian. Thus, this model can be generalized to systems with disorder as in [6], and then the hypothesis that the Fermi level is in a spectral gap of the Hamiltonian can be relaxed to the assumption that it is in a gap of extended states. This is a necessary step in order to establish the presence of plateaux.

In fact, this solves the apparent paradox that we still have a FQHE, even though the Hamiltonian $H_{\sigma, V}$ may not have any spectral gaps. The reason is that, as explained in [6], the domains of the cyclic 2-cocycles $\operatorname{tr}_{C}$ and $\operatorname{tr}_{K}$ are in fact larger than the smooth subalgebra $\mathcal{R}$. More precisely, there is a $*$-subalgebra $\mathcal{A}$ such that $\mathcal{R} \subset \mathcal{A} \subset \mathcal{U}(\Gamma, \sigma)$ and $\mathcal{A}$ is contained in the domains of $\operatorname{tr}_{C}$ and $\operatorname{tr}_{K}$. $\mathcal{A}$ is closed under the Besov space functional calculus, and the spectral projections $P_{E}$ of the Hamiltonian $H_{\sigma, V}$ that lie in $\mathcal{A}$ are called gaps in extended states. They include all the spectral projections onto gaps in the energy spectrum, but contain many more spectral projections. In particular, even though the Hamiltonian $H_{\sigma, V}$ may not have any spectral gaps, it may still have gaps in extended states. The results 
extend in a straightforward way to the case with disorder, where one allows the potential $V$ to be random, $c f$. [6].

Let us discuss the comparison with experimental data on the quantum Hall effect. Our model recovers the observed fractions (including the elusive 1/2). Table 1 below illustrates how low genus orbifolds with a small number of cone points are sufficient to recover many observed fractions. In this first table, we consider experimentally observed fractions, which we recover in our model. Notice how fractions like $1 / 3,2 / 5,2 / 3$, which experimentally appear with a wider and more clearly marked plateau, also correspond to the fractions realized by a larger number of orbifolds (we only checked the number of solutions for small values $\nu_{j} \leq 20, n=3$, $g=0$, and $\phi<1)$. These observations should be compared with the experimental data, cf. e.g. [29] 8 .

Regarding the varying width of the plateaux, what appears promising in Table 1 is the fact that the fractions that are more easily observed experimentally, i.e. those that appear with a larger and more clearly marked plateau (cf. e.g. [29, 8), also correspond to orbifold Euler characteristics that are realized by a large number of orbifolds. We can derive a corresponding qualitative graph of the widths, to be compared with the experimental ones. Table 2 shows how to obtain some experimentally observed fractions with $\phi>1$ (without counting multiplicities).

The main limitation of our model is that it seems to predict too many fractions, which at present do not seem to correspond to experimentally observed values. To our knowledge, however, this is also a limitation in the other theoretical models available in the literature. Another serious limitation is the fact that this model does not explain why even denominator fractions are more difficult to observe than odd ones. In fact, even for small number of cone points and low genus, one obtains a large number of orbifold Euler characteristics with even denominator, which are not justified experimentally. On the occurrence of even denominators in the fractional quantum Hall effect experiments, $c f . \quad 32$ [10 [15]. Table 3 provide a list of odd and even denominator fractions predicted by our model, using genus zero orbifolds with three cone points.

Questions and directions. We have discussed the transition from classical Bloch theory to noncommutative Bloch theory, as effect of the presence of a magnetic field. In particular, we have seen that the Brillouin zone becomes a noncommutative space. It would be interesting to investigate, using this point of view based on noncommutative geometry, what happens to the algebro-geometric theory of Fermi curves and periods. Another natural question related to the results discussed here is whether a Chern-Simons approach to the fractional quantum Hall effect may give a different justification for the presence of the orbifolds $\Sigma\left(g ; \nu_{1}, \ldots, \nu_{n}\right)$. In fact, these and their symmetric products appear as spaces of vortices in Chern-Simons (or Seiberg-Witten) theory.

Tables 1 and 2: experimental fractions. 


\begin{tabular}{|c|c|c|c|}
\hline experimental & $g=0 n=3$ & experimental & $g=0 n=3$ \\
\hline $1 / 3$ & $\begin{array}{c}(0 ; 3,6,6) \\
\Sigma(0 ; 4,4,6) \\
\Sigma(0 ; 3,4,12) \\
\Sigma(0 ; 2,12,12) \\
\Sigma(0 ; 2,10,15) \\
\Sigma(0 ; 2,9,18)\end{array}$ & $2 / 5$ & $\begin{array}{c}(0 ; 5,5,5) \\
\Sigma(0 ; 4,4,10) \\
\Sigma(0 ; 3,6,10) \\
\Sigma(0 ; 3,6,10) \\
\Sigma(0 ; 3,5,15) \\
\Sigma(0 ; 2,20,20)\end{array}$ \\
\hline $2 / 3$ & $\begin{array}{c}\Sigma(0 ; 9,9,9) \\
\Sigma(0 ; 8,8,12) \\
\Sigma(0 ; 6,12,12) \\
\Sigma(0 ; 6,10,15) \\
\Sigma(0 ; 6,9,18) \\
\Sigma(0 ; 5,15,15) \\
\Sigma(0 ; 5,12,20)\end{array}$ & $3 / 5$ & $\begin{array}{c}\Sigma(0 ; 5,10,10) \\
\Sigma(0 ; 6,6,15) \\
\Sigma(0 ; 4,12,15) \\
\Sigma(0 ; 4,10,20)\end{array}$ \\
\hline $4 / 9$ & $\begin{array}{c}\Sigma(0 ; 3,9,9) \\
\Sigma(0 ; 4,4,18) \\
\Sigma(0 ; 3,6,18)\end{array}$ & $5 / 9$ & $\begin{array}{c}\Sigma(0 ; 6,6,9) \\
\Sigma(0 ; 4,9,12) \\
\Sigma(0 ; 3,18,18)\end{array}$ \\
\hline $4 / 5$ & $\begin{array}{l}\Sigma(0 ; 15,15,15) \\
\Sigma(0 ; 12,15,20) \\
\Sigma(0 ; 10,20,20)\end{array}$ & $3 / 7$ & $\begin{array}{l}\Sigma(0 ; 4,4,14) \\
\Sigma(0 ; 3,6,14)\end{array}$ \\
\hline $4 / 7$ & $\Sigma(0 ; 7,7,7)$ & $5 / 7$ & $\Sigma(0 ; 7,14,14)$ \\
\hline experimental & $g=0$ or $g=1$ & & \\
\hline $8 / 5$ & $\Sigma(0 ; 2,4,4,5,5)$ & & \\
\hline $11 / 7$ & $\Sigma(0 ; 2,2,7,7,7)$ & & \\
\hline $14 / 9$ & $\Sigma(1 ; 3,9)$ & & \\
\hline $4 / 3$ & $\Sigma(1 ; 3,3)$ & & \\
\hline $7 / 5$ & $\Sigma(0 ; 5,5,10,10)$ & & \\
\hline $10 / 7$ & $\Sigma(0 ; 7,7,7,7)$ & & \\
\hline $13 / 9$ & $\Sigma(0 ; 6,6,9,9)$ & & \\
\hline $5 / 2$ & $\Sigma(1 ; 6,6,6)$ & & \\
\hline
\end{tabular}

Table 3: predicted fractions.

\begin{tabular}{|c||c||c||c|}
\hline odd & $g=0 n=3$ & even & $g=0 n=3$ \\
\hline \hline $8 / 15$ & $\Sigma(0 ; 5,6,10) \Sigma(0 ; 5,5,15)$ & $1 / 2$ & $\Sigma(0 ; 6,6,6) \Sigma(0 ; 5,5,10)$ \\
& $\Sigma(0 ; 4,6,20) \Sigma(0 ; 3,15,15)$ & & $\Sigma(0 ; 4,8,8) \Sigma(0 ; 4,6,12)$ \\
& $\Sigma(0 ; 3,12,20)$ & & $\Sigma(0 ; 4,5,20) \Sigma(0 ; 3,12,12)$ \\
& & & $\Sigma(0 ; 3,10,15) \Sigma(0 ; 3,9,18)$ \\
\hline $7 / 9$ & $\Sigma(0 ; 12,12,18) \Sigma(0 ; 10,15,18)$ & $1 / 4$ & $\Sigma(0 ; 4,4,4) \Sigma(0 ; 3,4,6)$ \\
& $\Sigma(0 ; 9,18,18)$ & & $\Sigma(0 ; 3,3,12) \Sigma(0 ; 2,8,8)$ \\
& & & $\Sigma(0 ; 3,3,12) \Sigma(0 ; 2,8,8)$ \\
& & & $\Sigma(0 ; 2,6,12) \Sigma(0 ; 2,5,20)$ \\
\hline $11 / 21$ & $\Sigma(0 ; 6,6,7) \Sigma(0 ; 4,7,12)$ & $7 / 12$ & $\Sigma(0 ; 6,8,8) \Sigma(0 ; 6,6,12)$ \\
& $\Sigma(0 ; 3,14,14)$ & & $\Sigma(0 ; 5,6,20) \Sigma(0 ; 4,12,12)$ \\
& & & $\Sigma(0 ; 4,10,15) \Sigma(0 ; 4,9,18)$ \\
\hline $16 / 21$ & $\Sigma(0 ; 12,12,14) \Sigma(0 ; 10,14,15)$ & & \\
& $\Sigma(0 ; 9,14,18)$ & & \\
\hline $11 / 15$ & $\Sigma(0 ; 10,10,15) \Sigma(0 ; 10,12,12)$ & & \\
& $\Sigma(0 ; 9,10,18) \Sigma(0 ; 6,20,20)$ & & \\
\hline
\end{tabular}




\section{References}

[1] J. Avron, R. Seiler, B. Simon, Charge deficiency, charge transport and comparison of dimensions, Comm. Math. Phys. Vol.159 (1994), no. 2, 399-422.

[2] J. Avron, R. Seiler, I. Yaffe, Adiabatic theorems and applications to the integer quantum Hall effect, Commun. Math. Phys. Vol.110 (1987) 33-49.

[3] J. Bellissard, A. van Elst, H. Schulz-Baldes, The noncommutative geometry of the quantum Hall effect, J.Math.Phys. 35 (1994) 5373-5451.

[4] J. Bellissard, The noncommutative geometry of aperiodic solids, in "Geometric and topological methods for quantum field theory (Villa de Leyva, 2001)", 86-156, World Scientific, 2003.

[5] A. Carey, K. Hannabuss, V. Mathai, P. McCann, Quantum Hall Effect on the hyperbolic plane, Commun. Math. Physics, Vol.190, no. 3 (1998) 629-673.

[6] A. Carey, K. Hannabuss, V. Mathai, Quantum Hall Effect on the Hyperbolic Plane in the presence of disorder, Letters in Mathematical Physics, Vol. 47 (1999) 215-236.

[7] A. Carey, K. Hannabuss, V. Mathai, Quantum Hall effect and noncommutative geometry, arXiv:math.OA/0008115

[8] T. Chakraborti, P. Pietilänen, The Quantum Hall Effects, Second Edition, Springer 1995.

[9] T.-S. Choy, J. Naset, J. Chen, S. Hershfield, and C. Stanton. A database of fermi surface in virtual reality modeling language (vrml), Bulletin of The American Physical Society, 45(1):L36 42, 2000 .

[10] R.G.Clark, R.J.Nicholas, A.Usher, C.T.Foxon, J.J.Harris, Surf.Sci. 170 (1986) 141.

[11] A. Connes, Non-commutative differential geometry, Publ.Math. IHES, Vol.62 (1985) 257360.

[12] A. Connes, Noncommutative geometry. Academic Press, Inc., San Diego, CA, 1994.

[13] A. Connes, H. Moscovici, Cyclic cohomology, the Novikov conjecture and hyperbolic groups, Topology, Vol. 29 (1990) no. 3, 345-388.

[14] J. Dodziuk, V. Mathai, S. Yates, Arithmetic properties of eigenvalues of generalized Harper operators on graphs, arXiv math.SP/0311315

[15] J.P.Eisenstein, G.S.Boebinger, L.N.Pfeiffer, K.W.West, S.He, Phys. Rev. Lett. 68 (1992) 1383; S.Q. Murphy, J.P.Eisenstein, G.S.Boebinger, L.N.Pfeiffer, K.W.West, Phys. Rev. Lett. 72 (1994) 728.

[16] D. Gieseker, H. Knörrer, E. Trubowitz, The geometry of algebraic Fermi curves, Perspectives in Mathematics, Vol.14. Academic Press, 1993. viii+236 pp.

[17] D. Gieseker, H. Knörrer, E. Trubowitz, An overview of the geometry of algebraic Fermi curves, in "Algebraic geometry: Sundance 1988", 19-46, Contemp. Math. Vol.116, Amer. Math. Soc. 1991.

[18] M. Gruber, Noncommutative Bloch theory, J.Math.Phys. Vol.42 (2001), no. 6, 2438-2465.

[19] E.H. Hall, On a new action of the magnet on electric currents, Amer. J. of Math. Vol.287, (1879) N.2.

[20] R. Ji, Smooth dense subalgebras of reduced group $C^{*}$-algebras, Schwartz cohomology of groups, and cyclic cohomology, J. Funct. Anal. 107 (1992), no. 1, 1-33.

[21] K. von Klitzing, G. Dorda, and M. Pepper, New method for high-accuracy determination of the fine-structure constant based on quantized hall resistance, Phys. Rev. Lett., Vol. 45 (1980) N.6, 494-497.

[22] Y. Kordyukov, V. Mathai and M.A. Shubin, Equivalence of spectral projections in semiclassical limit and a vanishing theorem for higher traces in K-theory, J.Reine Angew.Math. (Crelle), Vol.581 (2005) 44 pages (to appear).

[23] B. Laughlin, Quantized hall conductivity in two dimensions, Phys. Rev. B, Vol.23 (1981) 5232 .

[24] M. Marcolli and V. Mathai, Twisted index theory on good orbifolds, II: fractional quantum numbers, Communications in Mathematical Physics, Vol.217, no.1 (2001) 55-87.

[25] M. Marcolli and V. Mathai, Twisted index theory on good orbifolds, I: noncommutative Bloch theory, Communications in Contemporary Mathematics, Vol.1 (1999) 553-587.

[26] V. Mathai, On positivity of the Kadison constant and noncommutative Bloch theory, Tohoku Mathematical Publications, Vol.20 (2001) 107-124.

[27] V. Mathai, M. Shubin, Semiclassical asymptotics and gaps in the spectra of magnetic Schrödinger operators, Geometriae Dedicata, Vol. 91, no. 1, (2002) 155-173. 
[28] R.G.M $\cup \Phi$, The fractional quantum Hall effect, Chern-Simons theory, and integral lattices, in "Proceedings of the International Congress of Mathematicians", Vol. 1, 2 (Zrich, 1994), 75-105, Birkhäuser, 1995.

[29] H.L. Störmer, Advances in solid state physics, ed. P.Grosse, vol.24, Vieweg 1984.

[30] T. Sunada, A discrete analogue of periodic magnetic Schrödinger operators, Contemp. Math. Vol.173 (1994) 283-299.

[31] D.J. Thouless, M. Kohmono, M.P. Nightingale, M. den Nijs, Quantized Hall conductance in a two-dimensional periodic potential, Phys. Rev. Lett. 49 (1982) N.6, 405-408.

[32] R.Willett, J.P.Eisenstein, H.L.Störmer, D.C.Tsui, A.C.Gossard, J.H.English, Phys. Rev. Lett. 59 (1987) 1776.

[33] J. Xia, Geometric invariants of the quantum hall effect, Commun. Math. Phys. Vol. 119 (1988), 29-50.

M. Marcolli: Max-Planck Institut für Mathematik, Vivatsgasse 7, Bonn, D-53111 GerMANY

E-mail address: marcolli@mpim-bonn.mpg.de

V. Mathai: Department of Pure Mathematics, University of Adelaide, 5005 Australia

E-mail address: vmathai@maths.adelaide.edu.au 